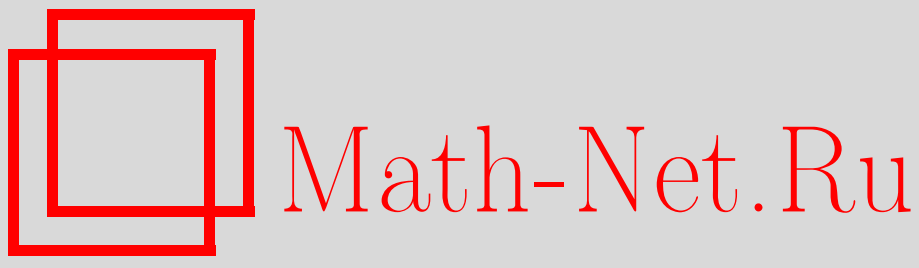

Г. В. Сандраков, Осреднение нестационарной системы Стокса с вязкостью в перфорированной области, Изв. РАН. Сер. матем., 1997, том 61, выпуск 1, 113-140

DOI: https://doi.org/10.4213/im107

Использование Общероссийского математического портала Math-Net.Ru подразумевает, что вы прочитали и согласны с пользовательским соглашением http://www . mathnet.ru/rus/agreement

Параметры загрузки:

IP : 54.80 .73 .141

26 апреля 2023 г., 16:49:54 
УДК 517.9

Г.В. Сандраков

\title{
Осреднение нестационарной системы Стокса с вязкостью в перфорированной области
}

\begin{abstract}
Доказаны утверждения о предельном поведении решений начально-краевой задачи для нестационарной системы уравнений Стокса в периодической перфорированной области с малым периодом $\varepsilon$. Предполагается, что коэффициент вязкости $\nu$ в этой системе является положительным параметром и удовлетворяет при $\varepsilon \rightarrow 0$ одному из следующих трех условий: $\nu / \varepsilon^{2} \rightarrow \infty, 1,0$. Рассмотрены также вырожденная система уравнений Стокса с нулевым коэффициентом вязкости и система уравнений Навье-Стокса в случае, когда коэффициент вязкости не является слишком малым.

Библиография: 22 наименования.
\end{abstract}

\section{§ 0. Введение}

В работе рассматривается нестационарная система уравнений Стокса в периодической перфорированной области с малым периодом $\varepsilon$, моделируюшая в линейном приближении течение вязкой несжимаемой жидкости в периодической пористой среде. Предполагается,что коэффициент вязкости $\nu$ в этой системе удовлетворяет при $\varepsilon \rightarrow 0$ одному из следующих трех условий:

$$
\begin{aligned}
& \nu / \varepsilon^{2} \rightarrow \infty \\
& \nu / \varepsilon^{2} \rightarrow 1 \\
& \nu / \varepsilon^{2} \rightarrow 0
\end{aligned}
$$

Будут получены осредненные (предельные) уравнения, вид которых зависит от предельного поведения коэффициента вязкости, и доказаны утверждения о сходимости в подходящих пространствах при $\varepsilon \rightarrow 0$ решений рассматриваемой системы к решениям осредненных уравнений.

Пусть для $m \geqslant 2$ вектор-функция $u_{\varepsilon}=\left(u_{1}^{\varepsilon}, \ldots, u_{m}^{\varepsilon}\right)$ и функция $p_{\varepsilon}$ являются решениями начально-краевой задачи для нестационарной системы уравнений Стокса с положительными параметрами $\varepsilon$ и $\nu$ :

$$
\begin{gathered}
\left(u_{\varepsilon}\right)_{t}^{\prime}-\nu \Delta u_{\varepsilon}+\nabla p_{\varepsilon}=f, \quad \operatorname{div} u_{\varepsilon}=0 \text { в } \Omega_{\varepsilon} \times(0, T), \\
u_{\varepsilon}=0 \text { на } \partial \Omega_{\varepsilon} \times(0, T),\left.\quad u_{\varepsilon}\right|_{t=0}=0 \text { в } \Omega_{\varepsilon},
\end{gathered}
$$

где $f \in L^{2}\left(0, T ; L^{2}(\Omega)^{m}\right)$ и положительное $T$ может быть произвольным. Зависящая от малого параметра $\varepsilon$ перфорированная область $\Omega_{\varepsilon}$ определяется следующим

Работа выполнена при финансовой поддержке Российского фонда фундаментальных исследований, грант 96-01-01394.

(C) Г.В. САНДРАКОВ 1997 
образом. Пусть $F_{1}$ является 1 -периодической связной подобластью $\mathbb{R}^{m}$ и $\Omega$ - ограниченная связная подобласть $\mathbb{R}^{m}$ с (локально) липшищевой гранищей. Тогда

$$
\Omega_{\varepsilon}=\Omega \cap F_{\varepsilon}
$$

где $F_{\varepsilon}=\varepsilon F_{1}=\left\{\varepsilon x, x=\left(x_{1}, \ldots, x_{m}\right) \in F_{1}\right\}$, и 1-периодичность означает периодичность множества $F_{1}$ с периодом 1 по каждому $x_{k}, k=1, \ldots, m$. Предполагается, что область $F_{1}$ допускает представление в виде объединения конечного числа 1-периодических областей с липшищевыми гранищами и справедливо неравенство Фридрихса

$$
\int_{F}|u|^{2} d x \leqslant C \int_{F}|\nabla u|^{2} d x \quad \forall u \in C^{\infty}(\bar{F}),\left.\quad u\right|_{S}=0,
$$

где $F=\square \cap F_{1}, S=\square \cap \partial F_{1}, \square=(0,1)^{m}$ - ячейка периодичности и $C$ - положительная постоянная. Предполагается также, что множество $F$ имеет положительную меру Лебега в $\mathbb{R}^{m}$. Отметим, что неравенство (0.6) заведомо выполнено, если множество $\square \backslash F$ имеет положительную меру Лебега в $\mathbb{R}^{m}$. В качестве примера двумерной $(m=2)$ области $F_{1}$, удовлетворяющей сформулированным условиям, можно выбрать 1-периодическое продолжение дополнения в $\bar{\square}$ к кругу с несколькими лучами (см. рисунок). Эта область $F_{1}$ не является 1 -периодической областью с липшицевой границей, но допускает представление в виде объединения конечного числа таких областей.

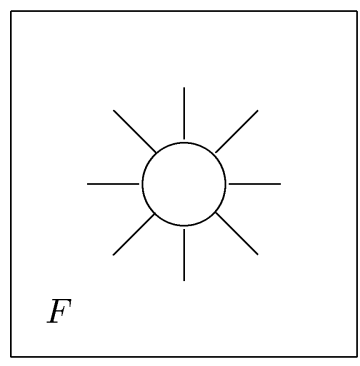

Известно, что для фиксированных $\varepsilon$ и $\nu$ существуют единственная вектор-функция $u_{\varepsilon}(t, x) \in L^{2}\left(0, T ; H_{0}^{1}\left(\Omega_{\varepsilon}\right)^{m}\right)$ и распределение $p_{\varepsilon}(t, x)$, определенное с точностью до произвольной функции от $t$, удовлетворяющие $(0.4)$ в соответствуюшем [1], [2] смысле. В дальнейшем предполагается, что вектор-функция $u_{\varepsilon}$ (соответствующая скорости жидкости в $\Omega_{\varepsilon}$ ) продолжена нулем на “твердую” фазу $\Omega \backslash \Omega_{\varepsilon}$ и через $\omega \subset \subset \Omega$ обозначается произвольная подобласть $\mathbb{R}^{m}$ с липшицевой гранищей, замыкание которой $\bar{\omega}$ принадлежит $\Omega$. Таким образом, для каждого $\varepsilon$ вектор скорости $u_{\varepsilon}$ определен в области $\Omega$, а $p_{\varepsilon}$ (соответствующее давлению жидкости) определено только на “жидкой” фазе $\Omega_{\varepsilon}$. Для каждого $\omega \subset \subset \Omega$ и достаточно малых $\varepsilon\left(\leqslant \varepsilon_{\omega}\right.$ для некоторого положительного $\left.\varepsilon_{\omega}\right)$ проверяется, что $p_{\varepsilon} \in L^{2}\left(0, T ; L^{2}\left(\omega \cap \Omega_{\varepsilon}\right) / \mathbb{R}\right)$ (определения используемых пространств см., например, в [1]).

Основные результаты работы составляют следующие теоремы о предельном поведении последовательностей $\left\{u_{\varepsilon}\right\}$ и $\left\{p_{\varepsilon}\right\}$ при $\varepsilon \rightarrow 0$ в предположении, что вьполнено одно из условий $(0.1)-(0.3)$. В этих теоремах через $n$ и $N$ обозначаются внешние нормали для $\partial \Omega$ и $S$, а через $E$ - единичная матрица для $\mathbb{R}^{m}$. Кроме того, через $\langle\cdot\rangle$ обозначается интеграл по $F$ от функций, определенных на этом множестве. 
ТЕОРема 1. Пусть выполнено условие (0.1). Тогда для каждого $\omega \subset \subset \Omega u$ достаточно малых є для последовательностей $\left\{u_{\varepsilon}\right\} u\left\{p_{\varepsilon}\right\}$ при $\varepsilon \rightarrow 0$ имеют место соотношения

$$
p_{\varepsilon} \rightarrow p \quad \text { в норме } \quad L^{2}\left(0, T ; L^{2}\left(\omega \cap \Omega_{\varepsilon}\right) / \mathbb{R}\right)
$$

$u$

$$
\left(\nu / \varepsilon^{2}\right) u_{\varepsilon} \rightarrow u=A_{1}(f-\nabla p) \quad \text { слабо в } \quad L^{2}\left(0, T ; L^{2}(\Omega)^{m}\right),
$$

где $\operatorname{div} u=0$ в $\Omega,(u, n)=0$ на $\partial \Omega$. Здесь $A_{1}=\langle a\rangle$ и а (y) является матричнозначным 1-периодическим решением краевой задачи для стационарной системь Стокса на F:

$$
-\Delta a+\nabla b=E, \quad \operatorname{div} a=0 \quad \text { в } \quad F, \quad a=0 \quad \text { нa } \quad S .
$$

ТЕОРема 2. Пусть выполнено условие (0.2). Тогда для каждого $\omega \subset \subset \Omega u$ достаточно малых в для последовательностей $\left\{u_{\varepsilon}\right\} u\left\{p_{\varepsilon}\right\}$ при $\varepsilon \rightarrow 0$ имеют место соотношения (0.7) и

$$
u_{\varepsilon} \rightarrow u=A_{2}(t) *(f-\nabla p) \quad \text { слабо в } \quad L^{2}\left(0, T ; L^{2}(\Omega)^{m}\right),
$$

где $\operatorname{div} u=0$ в $\Omega, \quad(u, n)=0$ на $\partial \Omega$ и mого, $u_{\varepsilon} \rightarrow$ u слабо- ${ }^{*}$ в $L^{\infty}\left(0, T ; L^{2}(\Omega)^{m}\right)$, если $f \in L^{1}\left(0, T ; L^{2}(\Omega)^{m}\right)$. Здесь $A_{2}(t)=\langle a(y, t)\rangle$ и а $(y, t)$ является матричнозначным 1-периодическим решением начально-краевой задачи для нестационарной системы Стокса на F:

$$
\begin{aligned}
& a_{t}^{\prime}-\Delta a+\nabla b=0, \quad \operatorname{div} a=0 \quad \text { в } F \times(0, \infty), \\
& a=0 \quad \text { на } S \times(0, \infty),\left.\quad a\right|_{t=0}=Q(E) \quad \text { в } F,
\end{aligned}
$$

где $Q(E)=E-\nabla B$ и $B(y)$ является векторнозначным 1-периодическим решением задачи Неймана на $F$ :

$$
\Delta B=0 \quad \text { в } \quad F, \quad(\nabla B, N)=(E, N) \quad \text { на } S .
$$

ТЕОРема 3. Пусть выполнено условие (0.3) и конечное $T$ фиксировано. Тогда для каждого $\omega \subset \subset \Omega$ и достаточно малых $\varepsilon$ для последовательностей $\left\{u_{\varepsilon}\right\} u\left\{p_{\varepsilon}\right\}$ при $\varepsilon \rightarrow 0$ имеют место соотношения (0.7) $и$

$$
u_{\varepsilon} \rightarrow u=A_{3} \int_{0}^{t}(f-\nabla p) d t \quad c л а б о-{ }^{*}{ }_{\theta} \quad L^{\infty}\left(0, T ; L^{2}(\Omega)^{m}\right)
$$

где $\operatorname{div} u=0$ в $\Omega, \quad(u, n)=0$ на $\partial \Omega$. Кроме того, $\left(u_{\varepsilon}\right)_{t}^{\prime} \rightarrow(u)_{t}^{\prime}$ слабо в $L^{2}\left(0, T ; L^{2}(\Omega)^{m}\right)$. Здесь $A_{3}=\langle E-\nabla B\rangle$ и $B(y)$ является решением краевой задачи (0.10). 
ЗАмечАниЕ 1. Можно показать, что в условиях теорем 1-3 соответствующие краевые задачи для "предельного" давления $p$ имеют единственные решения, принадлежащие $L^{2}\left(0, T ; H^{1}(\Omega) \cap L^{2}(\Omega) / \mathbb{R}\right)$. В случае выполнения условия $(0.1)$ соответствуюшая краевая задача для осредненного уравнения:

$$
\operatorname{div} A_{1}(f-\nabla p)=0 \quad \text { в } \Omega, \quad\left(A_{1}(f-\nabla p), n\right)=0 \quad \text { на } \partial \Omega,
$$

определяется как и для стационарной системы уравнений Стокса и зависит от времени только параметрически. В случае выполнения условия (0.3) осредненное уравнение имеет вид $(0.11)$ с $A_{1}$, замененным на $A_{3}$. Краевая задача для осредненного уравнения из теоремы 2 имеет схожую структуру:

$$
\operatorname{div} A_{2} *(f-\nabla p)=0 \quad \text { в } \Omega, \quad\left(A_{2} *(f-\nabla p), n\right)=0 \text { на } \partial \Omega,
$$

но зависит от времени нелокальньгм образом.

Аналог теоремы 3 справедлив также для нестационарной системы уравнений Стокса с нулевой вязкостью. Пусть в обозначениях, используемых для задачи (0.4), вектор-функция $v_{\varepsilon}$ и функция $q_{\varepsilon}$ являются решениями начально-краевой задачи для вырожденной нестационарной системы уравнений Стокса с положительным параметром $\varepsilon$ :

$$
\begin{gathered}
\left(v_{\varepsilon}\right)_{t}^{\prime}+\nabla q_{\varepsilon}=f, \quad \operatorname{div} v_{\varepsilon}=0 \text { в } \Omega_{\varepsilon} \times(0, T), \\
\left(v_{\varepsilon}, n_{\varepsilon}\right)=0 \text { на } \partial \Omega_{\varepsilon} \times(0, T),\left.\quad v_{\varepsilon}\right|_{t=0}=0 \text { в } \Omega_{\varepsilon},
\end{gathered}
$$

где $f \in L^{2}\left(0, T ; L^{2}(\Omega)^{m}\right) \cap L^{1}\left(0, T ; L^{2}(\Omega)^{m}\right)$ и $n_{\varepsilon}$ - внешняя нормаль для $\partial \Omega_{\varepsilon}$. Известно, что для фиксированного $\varepsilon$ существуют единственная вектор-функция $v_{\varepsilon}(t, x) \in L^{\infty}\left(0, T ; L^{2}\left(\Omega_{\varepsilon}\right)^{m}\right)$ и распределение $q_{\varepsilon}(t, x)$, определенное с точностью до произвольной функции от $t$, удовлетворяюшие (0.13) в соответствуюшем [2] смысле. Для каждого $\omega \subset \subset \Omega$ и достаточно малых $\varepsilon$ проверяется, что $q_{\varepsilon} \in$ $L^{2}\left(0, T ; L^{2}\left(\omega \cap \Omega_{\varepsilon}\right) / \mathbb{R}\right), \nabla q_{\varepsilon} \in L^{2}\left(0, T ; L^{2}\left(\Omega_{\varepsilon}\right)^{m}\right)$, и предполагается, что вектор скорости $v_{\varepsilon}$ продолжен нулем на “твердую" фазу $\Omega \backslash \Omega_{\varepsilon}$.

ТЕОРема 4. Для кажддого $\omega \subset \subset \Omega$ и достаточно мальх в для последовательностей $\left\{v_{\varepsilon}\right\}$ u $\left\{q_{\varepsilon}\right\}$ при $\varepsilon \rightarrow 0$ имеют место соотношения

$$
\begin{array}{ccc}
q_{\varepsilon} \rightarrow q \quad \text { в норме } & L^{2}\left(0, T ; L^{2}\left(\omega \cap \Omega_{\varepsilon}\right) / \mathbb{R}\right), \\
\nabla q_{\varepsilon} \rightarrow \nabla q \quad \text { в норме } & L^{2}\left(0, T ; L^{2}\left(\Omega_{\varepsilon}\right)^{m}\right), \\
v_{\varepsilon} \rightarrow v= & A_{3} \int_{0}^{t}(f-\nabla q) d t \quad \text { слабо- }{ }^{*}{ }_{\theta} \quad L^{\infty}\left(0, T ; L^{2}(\Omega)^{m}\right),
\end{array}
$$

где $\operatorname{div} v=0$ в $\Omega,(v, n)=0$ на $\partial \Omega$ и $A_{3}$ определено в теореме 3 . Кроме того, $\left(v_{\varepsilon}\right)_{t}^{\prime} \rightarrow(v)_{t}^{\prime}$ слабо в $L^{2}\left(0, T ; L^{2}(\Omega)^{m}\right)$.

Для системы уравнений (0.4) с неоднородным начальным условием также выполнены аналоги теорем $1-3$, но в более слабой форме. Для точной формулировки этих утверждений определим функцию $q_{\varepsilon}$ как решение краевой задачи Неймана $c$ положительным параметром $\varepsilon$ :

$$
\operatorname{div}\left(\nabla q_{\varepsilon}-w\right)=0 \quad \text { в } \quad \Omega_{\varepsilon}, \quad\left(\nabla q_{\varepsilon}-w, n_{\varepsilon}\right)=0 \text { на } \partial \Omega_{\varepsilon},
$$


где $w \in L^{2}(\Omega)^{m}$ и использованы обозначения, введенные для задач $(0.4)$ и (0.13). Известно [1], [2], что для фиксированного $\varepsilon$ существует единственное (с точностью до постоянной) решение задачи (0.14) с конечным интегралом Дирихле и равенство

$$
v_{\varepsilon}=w-\nabla q_{\varepsilon} \text { на } \Omega_{\varepsilon}
$$

определяет проекцию $w$ на $H\left(\Omega_{\varepsilon}\right)$ (подпространство соленоидальных вектор-функций из $L^{2}\left(\Omega_{\varepsilon}\right)^{m}$ с нулевой нормальной составляющей на $\left.\partial \Omega_{\varepsilon}\right)$. Пусть $u_{\varepsilon}$ и $p_{\varepsilon}$ являются решениями задачи (0.4) с неоднородным начальным условием:

$$
\left.u_{\varepsilon}\right|_{t=0}=v_{\varepsilon} \quad \text { в } \Omega_{\varepsilon} .
$$

Известно, что для фиксированных $\varepsilon$ и $\nu$ сушествуют единственная вектор-функция $u_{\varepsilon}(t, x) \in L^{2}\left(0, T ; H_{0}^{1}\left(\Omega_{\varepsilon}\right)^{m}\right)$ и распределение $p_{\varepsilon}(t, x)$, определенное с точностью до произвольной функции от $t$, удовлетворяющие $(0.4),(0.16)$ в соответствуюшем [1], [2] смысле. Для каждого $\omega \subset \subset \Omega$ и достаточно малых $\varepsilon$ проверяется, что $q_{\varepsilon} \in L^{2}\left(\omega \cap \Omega_{\varepsilon}\right) / \mathbb{R}, \nabla q_{\varepsilon} \in L^{2}\left(\Omega_{\varepsilon}\right)^{m}, \quad p_{\varepsilon} \in H^{-1}\left(0, T ; L^{2}(\omega \cap\right.$ $\left.\left.\Omega_{\varepsilon}\right) / \mathbb{R}\right)$, и предполагается, что вектор-функции $v_{\varepsilon}, u_{\varepsilon}$ продолжены нулем на "твердую" фазу $\Omega \backslash \Omega_{\varepsilon}$. Пространство $H^{-1}\left(0, T ; L^{2}\left(\omega \cap \Omega_{\varepsilon}\right) / \mathbb{R}\right)$ является сопряженным $H_{0}^{1}\left(0, T ; L^{2}\left(\omega \cap \Omega_{\varepsilon}\right) / \mathbb{R}\right)$ относительно скалярного произведения, продолженного с $L^{2}\left(0, T ; L^{2}\left(\omega \cap \Omega_{\varepsilon}\right) / \mathbb{R}\right)$, и наделяется естественной нормой.

Теорема 5. Пусть конечное Т фиксировано. Тогда для каждого $\omega \subset \subset \Omega$ и достаточно малых $\varepsilon$ для последовательностей $\left\{v_{\varepsilon}\right\},\left\{q_{\varepsilon}\right\},\left\{u_{\varepsilon}\right\} u\left\{p_{\varepsilon}\right\}$, удовлетворяющих (0.14), (0.15), (0.4) и (0.16), при $\varepsilon \rightarrow 0$ имеют место соотношения

$$
\begin{aligned}
& q_{\varepsilon} \rightarrow q \quad \text { в норме } \quad L^{2}\left(\omega \cap \Omega_{\varepsilon}\right) / \mathbb{R}, \quad \nabla q_{\varepsilon} \rightarrow \nabla q \quad \text { в норме } L^{2}\left(\Omega_{\varepsilon}\right)^{m}, \\
& v_{\varepsilon} \rightarrow v=A_{3}(w-\nabla q) \quad \text { слабо в } \quad L^{2}(\Omega)^{m},
\end{aligned}
$$

əде $\operatorname{div} v=0$ в $\Omega,(v, n)=0$ на $\partial \Omega$ и $A_{3}$ определено в теореме 3 ,

$$
p_{\varepsilon} \rightarrow p \quad \text { в норме } \quad H^{-1}\left(0, T ; L^{2}\left(\omega \cap \Omega_{\varepsilon}\right) / \mathbb{R}\right),
$$

и, кроме того:

(a) если выполнено условие (0.1), то

$$
\left(\nu / \varepsilon^{2}\right) u_{\varepsilon} \rightarrow A_{1}(f-\nabla p) \quad \text { в } \quad D^{\prime}(\Omega \times(0, T))^{m},
$$

где р является решением краевой задачи (0.11) и $A_{1}$ определено в теореме 1 ;

(b) если выполнено условие (0.2), то

$$
u_{\varepsilon} \rightarrow u=A_{2}(w-\nabla q)+A_{2} *(f-\nabla p) \quad c \bumpeq a 6 o^{*}{ }^{*} \quad \quad L^{\infty}\left(0, T ; L^{2}(\Omega)^{m}\right),
$$

где $\operatorname{div} u=0$ в $\Omega,(u, n)=0$ на $\partial \Omega$ и $A_{2}(t)$ определено в теореме 2 ;

(c) если выполнено условие (0.3), то

$$
u_{\varepsilon} \rightarrow A_{3}(w-\nabla q)+A_{3} \int_{0}^{t}(f-\nabla p) d t \quad \text { cлабо- }{ }^{*}{ }_{\theta} \quad L^{\infty}\left(0, T ; L^{2}(\Omega)^{m}\right),
$$

где р является решением краевой задачи (0.11) с $A_{1}$, замененным на $A_{3}$. 
ЗАмечАниЕ 2. Можно показать, что в условиях теоремы 5 соответствуюшая краевая задача для "предельного начального" давления $q$ имеет единственное решение, принадлежащее $H^{1}(\Omega) \cap L^{2}(\Omega) / \mathbb{R}$. Соответствующая краевая задача для "предельного" давления $p$ из теоремы $5,(\mathrm{~b})$ :

$$
\begin{aligned}
\operatorname{div} A_{2} *(f-\nabla p)+\operatorname{div} A_{2}(w-\nabla q) & =0 \quad \text { в } \quad \Omega, \\
\left(A_{2} *(f-\nabla p), n\right)+\left(A_{2}(w-\nabla q), n\right) & =0 \quad \text { на } \quad \partial \Omega,
\end{aligned}
$$

также имеет единственное решение, принадлежашее $L^{2}\left(0, T ; H^{1}(\Omega) \cap L^{2}(\Omega) / \mathbb{R}\right)$. В случае выполнения условий теоремы $5,($ a) в задаче $(0.4),(0.16)$ возникают погранслойные эффекты по времени, и поэтому сходимость (в смысле теории векторнозначных распределений на $\Omega \times(0, T))$ последовательности $\left\{\left(\nu / \varepsilon^{2}\right) u_{\varepsilon}\right\}$ достаточно слабая.

В случае, когда вязкость не слишком мала, аналог теоремы 1 выполнен также для трехмерной $(m=3)$ системы уравнений Навье-Стокса. Пусть в обозначениях, используемых для задачи $(0.4),(0.16)$, вектор-функция $u_{\varepsilon}$ и функция $p_{\varepsilon}$ являются решениями начально-краевой задачи для нестационарной системы уравнений Навье-Стокса с положительными параметрами $\varepsilon$ и $\nu$ :

$$
\begin{gathered}
\left(u_{\varepsilon}\right)_{t}^{\prime}-\nu \Delta u_{\varepsilon}+u_{\varepsilon} \nabla u_{\varepsilon}+\nabla p_{\varepsilon}=f, \quad \operatorname{div} u_{\varepsilon}=0 \quad \text { в } \quad \Omega_{\varepsilon} \times(0, T), \\
u_{\varepsilon}=0 \quad \text { на } \partial \Omega_{\varepsilon} \times(0, T),\left.\quad u_{\varepsilon}\right|_{t=0}=(\varepsilon / \sqrt{\nu}) v_{\varepsilon} \quad \text { в } \Omega_{\varepsilon},
\end{gathered}
$$

где $f \in L^{2}\left(0, T ; L^{2}(\Omega)^{3}\right)$. Известно, что для фиксированных $\varepsilon$ и $\nu$ существуют вектор-функция $u_{\varepsilon}(t, x) \in L^{2}\left(0, T ; H_{0}^{1}\left(\Omega_{\varepsilon}\right)^{3}\right)$ и распределение $p_{\varepsilon}(t, x)$, определенное с точностью до произвольной функции от $t$, удовлетворяюшие $(0.18)$ в соответствуюшем [1], [2] смысле. Проверяется, что решение $u_{\varepsilon}$ этой задачи определено однозначно, если выполнено условие

$$
\varepsilon^{3 / 2} / \nu^{2} \rightarrow 0
$$

Для каждого $\omega \subset \subset \Omega$ и достаточно малых $\varepsilon$ проверяется также, что $p_{\varepsilon} \in$ $H^{-1}\left(0, T ; L^{2}\left(\omega \cap \Omega_{\varepsilon}\right) / \mathbb{R}\right)$, и предполагается, что вектор скорости $u_{\varepsilon}$ продолжен нулем на “твердую" фазу $\Omega \backslash \Omega_{\varepsilon}$.

Теорема 6. Пусть выполнено условие (0.19) и конечное Т фиксировано. Тогда для каждого $\omega \subset \subset \Omega$ и достаточно малых $\varepsilon$ для последовательностей $\left\{u_{\varepsilon}\right\}$ u $\left\{p_{\varepsilon}\right\}$, удовлетворяющих (0.18), при $\varepsilon \rightarrow 0$ имеют место соотношения

$$
\begin{gathered}
p_{\varepsilon} \rightarrow p \quad \text { в норме } \\
\left(\nu / \varepsilon^{2}\right) u_{\varepsilon} \rightarrow A_{1}(f-\nabla p)
\end{gathered}
$$

әде р является решением краевой задачи (0.11).

Пусть в обозначениях, используемых для задачи (0.4), вектор-функция $u_{\varepsilon}$ и функция $p_{\varepsilon}$ являются решениями краевой задачи для трехмерной стационарной системы уравнений Навье-Стокса с положительными параметрами $\varepsilon$ и $\nu$ :

$$
-\nu \Delta u_{\varepsilon}+u_{\varepsilon} \nabla u_{\varepsilon}+\nabla p_{\varepsilon}=f, \quad \operatorname{div} u_{\varepsilon}=0 \quad \text { в } \quad \Omega_{\varepsilon}, \quad u_{\varepsilon}=0 \text { на } \partial \Omega_{\varepsilon},
$$


где $f \in L^{2}(\Omega)^{3}$. Известно, что для фиксированных $\varepsilon$ и $\nu$ сушествуют решение $u_{\varepsilon}(x) \in H_{0}^{1}\left(\Omega_{\varepsilon}\right)^{3}$ и распределение $p_{\varepsilon}(x)$, определенное с точностью до произвольной постоянной, удовлетворяющие (0.20) в соответствующем [1], [2] смысле. Можно показать, что решение $u_{\varepsilon}$ этой задачи определено однозначно, если выполнено условие (0.19). Для каждого $\omega \subset \subset \Omega$ и достаточно малых $\varepsilon$ проверяется также, что $p_{\varepsilon} \in L^{2}\left(\omega \cap \Omega_{\varepsilon}\right) / \mathbb{R}$, и предполагается, что вектор скорости $u_{\varepsilon}$ продолжен нулем на “твердую” фазу $\Omega \backslash \Omega_{\varepsilon}$.

ТЕОРема 7. Пусть выполнено условие (0.19). Тогда для каждого $\omega \subset \subset \Omega$ и достаточно малых є для последовательностей $\left\{u_{\varepsilon}\right\}$ u $\left\{p_{\varepsilon}\right\}$, удовлетворяющих (0.20), при $\varepsilon \rightarrow 0$ имеют место соотношения

$p_{\varepsilon} \rightarrow p \quad$ в норме $L^{2}\left(\omega \cap \Omega_{\varepsilon}\right) / \mathbb{R}, \quad\left(\nu / \varepsilon^{2}\right) u_{\varepsilon} \rightarrow A_{1}(f-\nabla p)$ слабо в $L^{2}(\Omega)^{3}$, әде р является решением краевой задачи (0.11).

ЗАмечаниЕ 3. Для двумерных задач (0.18) и (0.20) теоремы 6 и 7 также выполнены, если в условии (0.19) величину $\varepsilon^{3 / 2} / \nu^{2}$ заменить на $\varepsilon / \nu$.

Доказательства представленных здесь утверждений даны в $\S 2$. Несколько лемм, используемых в этих доказательствах, приведены в $\S 1$. В дальнейшем изложении $C$ обозначает постоянные, не зависящие от $\varepsilon$ и $\nu$, а функции, определенные на $\Omega_{\varepsilon}$ и обнуляюшиеся на $\partial \Omega_{\varepsilon}$, считаются, как правило, продолженными нулем на “твердую” фазу $\Omega \backslash \Omega_{\varepsilon}$.

ЗАмЕчАниЕ 4. Вопросы осреднения стационарной системы уравнений Стокса в периодических перфорированных областях рассматривались в работах [3]-[6]. Для доказательства утверждений о сходимости в [3], [4] использовался технически сложный метод продолжения давлений, предложенный Л. Тартаром. В статье [6] предложен новый метод, не использующий такого продолжения и позволяющий рассматривать наиболее общие периодические перфорированные области. Метод продолжения давлений применялся также при изучении вопросов осреднения нестационарной системы уравнений Навье-Стокса в работе [7] и нестационарной системы уравнений Стокса с вязкостью порядка $\varepsilon^{2}$ в работах [8], [9]. Условия

(0.1)-(0.3) возникали ранее в [10] при осреднении линеаризованной системы уравнений гидродинамики с периодическими коэффициентами и малой вязкостью. Для доказательства приведенных здесь утверждений о сходимости будут использованы методы, разработанные в работах [6] и [10]. Некоторые из этих утверждений были анонсированы в заметке [11], где также применялся метод продолжения давлений. Использование здесь метода В. В. Жикова позволило значительно ослабить требования из [11] на рассматриваемые перфорированные области. Кроме того, следуя [6], из соотношения (0.7) несложно вывести существование продолжения (на область $\Omega$ ) давления $p_{\varepsilon}$, сходяшегося при $\varepsilon \rightarrow 0$ к "предельному" давлению $p$ в норме $L^{2}\left(0, T ; L^{2}(\omega) / \mathbb{R}\right)$.

Автор выражает признательность Н. С. Бахвалову и В. В. Жикову за многочисленные полезные советы по рассматриваемым в статье вопросам.

\section{$\S 1$. Леммы}

ЛЕмма 1. Для $u_{\varepsilon} \in L^{2}\left(0, T ; H_{0}^{1}\left(\Omega_{\varepsilon}\right)\right)$ выполнено неравенство

$$
\|u\|_{L^{2}\left(0, T ; L^{2}(\Omega)\right)} \leqslant \varepsilon C\|\nabla u\|_{L^{2}\left(0, T ; L^{2}(\Omega)\right)} \text {. }
$$


ДокаЗАТЕЛЬСТво. Для П.в. $t$ (почти всех $t \in(0, T))$ функция $u(t)$ является элементом пространства $H_{0}^{1}\left(\Omega_{\varepsilon}\right)$. Следствием предположения $(0.6)$ является (см., например, [3], [12]) оценка

$$
\|v\|_{L^{2}(\Omega)} \leqslant \varepsilon C\|\nabla v\|_{L^{2}(\Omega)} \quad \forall v \in H_{0}^{1}\left(\Omega_{\varepsilon}\right) .
$$

Напомним, что функции из $H_{0}^{1}\left(\Omega_{\varepsilon}\right)$ продолжаются нулем на $\Omega \backslash \Omega_{\varepsilon}$. Таким образом, $\|u(t)\|_{L^{2}(\Omega)}^{2} \leqslant \varepsilon^{2} C\|\nabla u(t)\|_{L^{2}(\Omega)}^{2}$ для п.в. $t$, и интегрирование этого неравенства по $(0, T)$ завершает доказательство леммы 1 .

ЛЕмма 2. Пусть распределение $p(x, t)$ определено на $\Omega_{\varepsilon} \times(0, T)$ и удовлетворяет условию $\nabla_{x} p \in L^{2}\left(0, T ; H^{-1}\left(\Omega_{\varepsilon}\right)^{m}\right)$. Тогда для каждого $\omega \subset \subset \Omega u$ достаточно мальх $\varepsilon$ выполнено неравенство

$$
\|p\|_{\left.L^{2}\left(0, T ; L^{2}\left(\omega \cap \Omega_{\varepsilon}\right) / \mathbb{R}\right)\right)} \leqslant \varepsilon^{-1} C\|\nabla p\|_{L^{2}\left(0, T ; H^{-1}\left(\Omega_{\varepsilon}\right)^{m}\right)} .
$$

ДоКАЗАТЕЛЬСтво. Для распределений $g(x)$, определенных на $\Omega_{\varepsilon}$ и удовлетворяюших условию $\nabla g \in H^{-1}\left(\Omega_{\varepsilon}\right)^{m}$, в работе [6] доказана следуюшая важная оценка: для каждого $\omega \subset \subset \Omega$ и достаточно малых $\varepsilon$

$$
\|q\|_{L^{2}\left(\omega \cap \Omega_{\varepsilon}\right) / \mathbb{R}} \leqslant \varepsilon^{-1} C\|\nabla q\|_{H^{-1}\left(\Omega_{\varepsilon}\right)^{m}} .
$$

Отметим, что для фиксированных $\omega$ и (достаточно малых) $\varepsilon$ включение $q \in$ $L^{2}\left(\omega \cap \Omega_{\varepsilon}\right) / \mathbb{R}$ следует из неравенства, доказанного в работе [13] (в случае, когда область $F_{1}$ липшицева, это включение следует также из [1, предложение 1.2]). Однако соответствуюшая константа в неравенстве из [13] зависит от $\varepsilon$, и явньй вид этой зависимости установлен в работе [6]. Таким образом, $\|p(t)\|_{L^{2}\left(\omega \cap \Omega_{\varepsilon}\right) / \mathbb{R}}^{2} \leqslant$ $\varepsilon^{-2} C\|\nabla p(t)\|_{H^{-1}\left(\Omega_{\varepsilon}\right)^{m}}^{2}$ для п.в. $t$, и интегрирование этого неравенства по $(0, T)$ завершает доказательство леммы 2.

ЛЕмма 3. Для $u_{\varepsilon} \in L^{2}\left(0, T ; L^{2}\left(\Omega_{\varepsilon}\right)\right)$ выполнено неравенство

$$
\|u\|_{L^{2}\left(0, T ; H^{-1}\left(\Omega_{\varepsilon}\right)\right)} \leqslant \varepsilon C\|u\|_{L^{2}\left(0, T ; L^{2}\left(\Omega_{\varepsilon}\right)\right)} .
$$

ДоКАЗАТЕЛЬСТво. Пусть $v \in L^{2}\left(\Omega_{\varepsilon}\right)$ и $(\cdot, \cdot)_{\varepsilon}$ обозначает скалярное произведение между элементами пространств $H_{0}^{1}\left(\Omega_{\varepsilon}\right)$ и $H^{-1}\left(\Omega_{\varepsilon}\right)$. Тогда

$$
\|v\|_{H^{-1}\left(\Omega_{\varepsilon}\right)}^{2}=\left((-\Delta)^{-1} v, v\right)_{\varepsilon}
$$

где $(-\Delta)^{-1} v=\widetilde{v}$ является решением задачи Дирихле

$$
-\Delta \widetilde{v}=v \text { в } \Omega_{\varepsilon}, \quad \widetilde{v}=0 \text { на } \partial \Omega_{\varepsilon}, \quad \widetilde{v} \in H_{0}^{1}\left(\Omega_{\varepsilon}\right) .
$$

Для решения этой задачи из предположения (0.6) следует (см., например, [12]) оценка $\|\widetilde{v}\|_{H_{0}^{1}\left(\Omega_{\varepsilon}\right)} \leqslant \varepsilon C\|v\|_{L^{2}\left(\Omega_{\varepsilon}\right)}$, и поэтому

$$
\|v\|_{H^{-1}\left(\Omega_{\varepsilon}\right)}^{2} \leqslant\|\widetilde{v}\|_{H_{0}^{1}\left(\Omega_{\varepsilon}\right)}\|v\|_{H^{-1}\left(\Omega_{\varepsilon}\right)} \leqslant \varepsilon C\|v\|_{L^{2}\left(\Omega_{\varepsilon}\right)}\|v\|_{H^{-1}\left(\Omega_{\varepsilon}\right)} .
$$

Таким образом, $\|u(t)\|_{H^{-1}\left(\Omega_{\varepsilon}\right)}^{2} \leqslant \varepsilon^{2} C\|u(t)\|_{L^{2}\left(\Omega_{\varepsilon}\right)}^{2}$ для п.в. $t$, и интегрирование этого неравенства по $(0, T)$ завершает доказательство леммы 3 .

Отметим, что для $v \in L^{2}\left(0, T ; H_{0}^{1}\left(\Omega_{\varepsilon}\right)\right)$ непосредственно из определений следует равенство

$$
\|\Delta u\|_{L^{2}\left(0, T ; H^{-1}\left(\Omega_{\varepsilon}\right)\right)}=\|\nabla u\|_{L^{2}\left(0, T ; L^{2}\left(\Omega_{\varepsilon}\right)^{m}\right)} .
$$


ЛЕмма 4. Для решений задачи (0.4) выполненьи неравенства

$$
\begin{aligned}
\nu \varepsilon^{-1}\left\|\nabla u_{\varepsilon}\right\|_{L^{2}\left(0, T ; L^{2}(\Omega)^{m \times m}\right)} & \leqslant C\|f\|_{L^{2}\left(0, T ; L^{2}(\Omega)\right)}, \\
\nu \varepsilon^{-2}\left\|u_{\varepsilon}\right\|_{L^{2}\left(0, T ; L^{2}(\Omega)^{m}\right)} & \leqslant C\|f\|_{L^{2}\left(0, T ; L^{2}(\Omega)\right)}, \\
\left\|\left(u_{\varepsilon}\right)_{t}^{\prime}\right\|_{L^{2}\left(0, T ; L^{2}(\Omega)^{m}\right)} & \leqslant C\|f\|_{L^{2}\left(0, T ; L^{2}(\Omega)\right)}, \\
\varepsilon^{-1}\left\|\nabla p_{\varepsilon}\right\|_{L^{2}\left(0, T ; H^{-1}\left(\Omega_{\varepsilon}\right)^{m}\right)} & \leqslant C\|f\|_{L^{2}\left(0, T ; L^{2}(\Omega)\right)} .
\end{aligned}
$$

Кроме того, если $f \in L^{1}\left(0, T ; L^{2}(\Omega)^{m}\right)$, то для п.в. $t$ выполнено неравенство

$$
\left\|u_{\varepsilon}\right\|_{L^{2}(\Omega)^{m}} \leqslant\|f\|_{L^{1}\left(0, T ; L^{2}(\Omega)^{m}\right)} .
$$

ДокАЗАТЕльСтво. Умножая скалярно в $L^{2}\left(\Omega_{\varepsilon}\right)^{m}$ первое уравнение $(0.4)$ на $u_{\varepsilon}$, получаем

$$
\left(\left\|u_{\varepsilon}\right\|_{L^{2}\left(\Omega_{\varepsilon}\right)^{m}}^{2}\right)_{t}^{\prime}+2 \nu\left\|\nabla u_{\varepsilon}\right\|_{L^{2}\left(\Omega_{\varepsilon}\right)^{m \times m}}^{2}=2\left(f, u_{\varepsilon}\right)_{L^{2}\left(\Omega_{\varepsilon}\right)^{m}} .
$$

Интегрируя это равенство по $(0, T)$ и используя лемму 1 , имеем

$$
\begin{aligned}
& \nu\left\|\nabla u_{\varepsilon}\right\|_{L^{2}\left(0, T ; L^{2}\left(\Omega_{\varepsilon}\right)^{m \times m}\right)}^{2} \leqslant \int_{0}^{T}\left(f, u_{\varepsilon}\right)_{L^{2}\left(\Omega_{\varepsilon}\right)^{m}} d t \\
& \quad \leqslant\|f\|_{L^{2}\left(0, T ; L^{2}\left(\Omega_{\varepsilon}\right)^{m}\right)}\left\|u_{\varepsilon}\right\|_{L^{2}\left(0, T ; L^{2}\left(\Omega_{\varepsilon}\right)^{m}\right)} \\
& \quad \leqslant \varepsilon C\|f\|_{L^{2}\left(0, T ; L^{2}(\Omega)^{m}\right)}\left\|\nabla u_{\varepsilon}\right\|_{L^{2}\left(0, T ; L^{2}\left(\Omega_{\varepsilon}\right)^{m \times m}\right)}
\end{aligned}
$$

что доказывает первое и второе неравенства леммы. Третье неравенство доказывается аналогично с помощью умножения первого уравнения $(0.4)$ на $\left(u_{\varepsilon}\right)_{t}^{\prime}$. Учитывая эти неравенства, (1.1) и лемму 3 , заключаем, что

$$
\begin{gathered}
\left\|\nabla p_{\varepsilon}\right\|_{L^{2}\left(0, T ; H^{-1}\left(\Omega_{\varepsilon}\right)^{m}\right)}=\left\|f+\nu \Delta u_{\varepsilon}-\left(u_{\varepsilon}\right)_{t}^{\prime}\right\|_{L^{2}\left(0, T ; H^{-1}\left(\Omega_{\varepsilon}\right)^{m}\right)} \\
\leqslant\|f\|_{L^{2}\left(0, T ; H^{-1}\left(\Omega_{\varepsilon}\right)^{m}\right)}+\nu\left\|\nabla u_{\varepsilon}\right\|_{L^{2}\left(0, T ; L^{2}\left(\Omega_{\varepsilon}\right)^{m \times m}\right)} \\
+\left\|\left(u_{\varepsilon}\right)_{t}^{\prime}\right\|_{L^{2}\left(0, T ; H^{-1}\left(\Omega_{\varepsilon}\right)^{m}\right)} \leqslant \varepsilon C\|f\|_{L^{2}\left(0, T ; L^{2}\left(\Omega_{\varepsilon}\right)^{m}\right)} .
\end{gathered}
$$

Кроме того, из $(1.2)$ следует неравенство $\left(\left\|u_{\varepsilon}\right\|_{L^{2}\left(\Omega_{\varepsilon}\right)^{m}}\right)_{t}^{\prime} \leqslant\|f\|_{L^{2}\left(\Omega_{\varepsilon}\right)^{m}}$, и интегрирование этого неравенства по $(0, t)$ завершает доказательство леммы 4 .

Обозначим через $H_{P}^{1}(F, S)$ замыкание в $H^{1}(F)$ множества $C_{P}^{\infty}(F, S)$ - гладких 1-периодических функций, обнуляюшихся вблизи $S$, а через $H_{P}^{-1}(F, S)$ - пространство, сопряженное $H_{P}^{1}(F, S)$ относительно скалярного произведения, продолженного с $L^{2}(F)$. Определим также множества вектор-функций

$$
\begin{aligned}
& H(F)=\left\{a \in L^{2}(F)^{m}: \operatorname{div} a=0 \quad \text { в } \quad F, \quad(a, N)=0 \text { на } S\right\}, \\
& H(\Omega)=\left\{u \in L^{2}(\Omega)^{m}: \operatorname{div} u=0 \quad \text { в } \quad \Omega, \quad(u, n)=0 \text { на } \partial \Omega\right\},
\end{aligned}
$$

являюшиеся (см., например, [1]) замкнутыми подпространствами в $L^{2}(F)^{m}$ и $L^{2}(\Omega)^{m}$ соответственно.

Следующее утверждение следует из результатов работы [13] (в случае, когда область $F_{1}$ липшицева, это утверждение следует также из $[1$, предложение 1.2 , лемма 6.1]). 
Лемма 5. Нормы $\|\cdot\|_{L^{2}(F) / \mathbb{R}} u\|\nabla(\cdot)\|_{H_{P}^{-1}(F, S)}$ на пространстве $L^{2}(F) / \mathbb{R}$ әквивалентны.

Лемма 6. Пусть $a \in H(F)$. Тогда найдется последовательность $\left\{a_{k}\right\} \subset$ $C_{P}^{\infty}(F, S)^{m}$ соленоидальньх $\left(\operatorname{div} a_{k}=0\right.$ в $\left.F\right)$ вектор-функций, сходящаяся $к а$ в норме $L^{2}(F)^{m}$.

ДокАЗАТЕЛЬСтво. Это утверждение следует из леммы 5 и результатов, доказанных в [1, теорема 1.4].

Лемма 7. Пусть $u \in L^{2}(0, T ; H(\Omega))$. Тогда найдется последовательность $\left\{u_{k}\right\} \subset C_{0}^{\infty}((0, T) \times \Omega)^{m}$ соленоидальных $\left(\operatorname{div} u_{k}=0\right.$ в $\left.\Omega\right)$ вектор-функиий, сходящаяся $\kappa$ и в норме $L^{2}\left(0, T ; L^{2}(\Omega)^{m}\right)$.

ДокАЗАТЕЛЬСТво. Известно [1, теорема 1.4$]$, что в $H(\Omega)$ плотно множество $V(\Omega)$ соленоидальных вектор-функций из $C_{0}^{\infty}(\Omega)^{m}$. Пространство $H(\Omega)$ является замкнутым подпространством сепарабельного пространства $L^{2}(\Omega)^{m}$. Следовательно, найдется последовательность $\left\{\varphi_{k}\right\} \subset V(\Omega)$ таких вектор-функций, образуюшая ортонормированный базис в $H(\Omega)$. Аналогично, найдется последовательность $\left\{a_{s}\right\} \subset C_{0}^{\infty}(0, T)$, образуюшая ортонормированный базис в $L^{2}(0, T)$. Утверждение из $[14$, гл. $2, \S 4]$ о том, что конечные линейные комбинации векторов вида $a_{s} \varphi_{k}$ образуют плотное подмножество в $L^{2}(0, T ; H(\Omega))$, завершает доказательство леммы 7 .

ЛЕмма 8. Пусть $a \in L^{2}(F) / \mathbb{R}$. Тогда найдется такое $w \in H_{P}^{1}(F, S)^{m}$, ито $\operatorname{div} w=a u$

$$
\|w\|_{H_{P}^{1}(F, S)^{m}} \leqslant C\|a\|_{L^{2}(F) / \mathbb{R}} \cdot
$$

ДокАЗАТЕльСтво. В случае, когда область $F_{1}$ имеет границу класса $C^{2}$, это утверждение доказано в $[5$, гл. $4, \S 7]$. С учетом результатов работы [15] общий случай рассматривается аналогичным образом.

Следуя [16], определим пространство $E_{0}\left(C^{+} ; L^{2}(\Omega)\right)$ как множество функций $U(\sigma)=U(\alpha+i \beta)$ со значениями в $L^{2}(\Omega)$, заданных и голоморфных в комплексной полуплоскости $C^{+}=\{\sigma \in C: \sigma=\alpha+i \beta, \alpha>0\}$, для которых конечна величина

$$
\|U(\sigma)\|_{E_{0}\left(C^{+} ; L^{2}(\Omega)\right)}^{2}=\int_{-\infty}^{\infty}\|U(i \beta)\|_{L^{2}(\Omega)}^{2} d \beta,
$$

где интеграл определяется как предел в среднем интеграла от $K$ до $-K$ при $K \rightarrow \infty$. Последнее равенство определяет норму в пространстве $E_{0}\left(C^{+} ; L^{2}(\Omega)\right)$.

Следуюший вариант теоремы Пэли-Винера доказан в [16].

Теорема 9. Преобразование Лапласа

$$
\widehat{u(t)}=\int_{0}^{\infty} e^{-\sigma t} u(t) d t=U(\sigma)
$$

отображает пространство $L^{2}\left(0, \infty ; L^{2}(\Omega)\right)$ на пространство $E_{0}\left(C^{+} ; L^{2}(\Omega)\right)$ взаимно однозначно и взаимно непрерывно.

Аналогично определяются пространства $E_{0}\left(C^{+} ; H_{0}^{1}(\Omega)\right)$ и $E_{0}\left(C^{+} ; L^{2}(F)\right)$, для которых также вьполнен аналог теоремы 9 . Кроме того [16], преобразование Лапласа коммутирует с дифференцированиями по пространственным переменным и оператор свертки по $t$ переводится преобразованием Лапласа в оператор поточечного умножения. 


\section{§2. Доказательства}

ДОКАЗАТЕЛЬСТВО ТЕОРЕМЫ 1. В СоответствиИ с результатами [1] и леммой 5 решение $a(y), b(y)$ задачи $(0.8)$ с компонентами из $H_{P}^{1}(F, S), L^{2}(F) / \mathbb{R}$ существует и единственно. Определим $a_{\varepsilon}(x)=a(x / \varepsilon),\left(\nabla_{y} a\right)_{\varepsilon}(x)=\left(\nabla_{y} a\right)(x / \varepsilon), b_{\varepsilon}(x)=b(x / \varepsilon)$ и продолжим эти функции по $\varepsilon$-периодичности на $\Omega_{\varepsilon}$ (точнее, продолжим, например, $a(x / \varepsilon)$ как $\varepsilon$-периодическую функцию на $F_{\varepsilon}$ и возьмем ограничение полученной функции на $\Omega_{\varepsilon} \subset F_{\varepsilon}$ ) и $a_{\varepsilon}$ нулем на $\Omega \backslash \Omega_{\varepsilon}$. Тогда (см., например, [3], [4])

$$
\left\|a_{\varepsilon}\right\|_{L^{2}(\Omega)^{m \times m}} \leqslant C, \quad\left\|\left(\nabla_{y} a\right)_{\varepsilon}\right\|_{L^{2}(\Omega)^{m \times m \times m}} \leqslant C, \quad\left\|b_{\varepsilon}\right\|_{L^{2}\left(\Omega_{\varepsilon}\right)^{m}} \leqslant C .
$$

Известно [3], что $A_{1}=\langle a\rangle$ является симметричной положительно определенной матрицей с постоянными компонентами. Кроме того [10], найдется такое $W_{\varepsilon}(x) \in$ $H^{1}(\Omega)^{m \times m \times m}$, что $\left\|W_{\varepsilon}\right\|_{L^{2}(\Omega)^{m \times m \times m}} \leqslant C$ и

$$
a_{\varepsilon}-A_{1}=\varepsilon \operatorname{div}\left(W_{\varepsilon}\right)
$$

Для доказательства этого утверждения достаточно выбрать $W(y)=$ $\nabla_{y} \Delta_{y}^{-1}\left(a-A_{1}\right)$, продолжить функцию $W(x / \varepsilon)$ по $\varepsilon$-периодичности на $\Omega$ и положить $W_{\varepsilon}(x)=W(x / \varepsilon)$. Здесь $\widetilde{a}=\Delta_{y}^{-1}\left(a-A_{1}\right)$ обозначает 1-периодическое решение задачи $\Delta_{y} \widetilde{a}=a-A_{1}$ (подробно изученной, например, в [5]).

По предположению мера Лебега $|F|$ множества $F$ положительна, и поэтому $A_{1} /|F|-a \in\left(L^{2}(F) / \mathbb{R}\right)^{m \times m}$. В силу леммы 8 найдется такое $w(y) \in$ $H_{P}^{1}(F, S)^{m \times m \times m}$, что $\operatorname{div}_{y} w=A_{1} /|F|-a$. По определению найдется последовательность $\left\{w^{s}(y)\right\} \subset C_{P}^{\infty}(F, S)^{m \times m \times m}$, сходяшаяся к $w(y)$ в норме $H_{P}^{1}(F, S)^{m \times m \times m}$. Определим $w_{\varepsilon}^{s}(x)=w^{s}(x / \varepsilon),\left(\operatorname{div}_{y} w^{s}\right)_{\varepsilon}(x)=\left(\operatorname{div}_{y} w^{s}\right)(x / \varepsilon)$, продолжим эти функции по $\varepsilon$-периодичности на $\Omega_{\varepsilon}$ и $w_{\varepsilon}$ нулем на $\Omega \backslash \Omega_{\varepsilon}$. Из ограниченности области $\Omega_{\varepsilon}$ следует, что равномерно по $\varepsilon$ выполнено соотношение

$$
\begin{aligned}
& \left\|\left(\operatorname{div}_{y} w^{s}\right)_{\varepsilon}-A_{1} /|F|+a_{\varepsilon}\right\|_{L^{2}\left(\Omega_{\varepsilon}\right)^{m \times m}} \\
& \quad \leqslant C\left\|\operatorname{div}_{y} w^{s}(y)-A_{1} /|F|+a(y)\right\|_{L^{2}(F)^{m \times m}} \rightarrow 0 \text { при } s \rightarrow \infty .
\end{aligned}
$$

Матрица $A_{1}$ положительно определена, и поэтому осредненное уравнение $(0.11)$ имеет для п.в. $t$ единственное решение $p(t) \in H^{1}(\Omega) \cap L^{2}(\Omega) / \mathbb{R}$, удовлетворяюшее соответствуюшей априорной оценке (см., например, [12]). Интегрирование этой оценки по $(0, T)$ приводит к неравенству

$$
\|p\|_{L^{2}\left(0, T ; L^{2}(\Omega) / \mathbb{R}\right)}+\|\nabla p\|_{L^{2}\left(0, T ; L^{2}(\Omega)^{m}\right)} \leqslant C\|f\|_{L^{2}\left(0, T ; L^{2}(\Omega)\right)} .
$$

Пусть $G=A_{1}(f-\nabla p)$. Тогда $\operatorname{div} G=0$ в $\Omega$ и $(G, n)=0$ на $\partial \Omega$. В силу леммы 7 найдется последовательность $\left\{G_{k}\right\} \subset C_{0}^{\infty}((0, T) \times \Omega)^{m}$ соленоидальных вектор-функций, сходящаяся к $G$ в норме $L^{2}\left(0, T ; L^{2}(\Omega)^{m}\right)$. Пусть $P_{k}=A_{1}^{-1} G_{k}$. Тогда

$$
\operatorname{div} A_{1} P_{k}=A_{1} \nabla P_{k}=0 .
$$

Вновь учитьвая положительную определенность матрицы $A_{1}$, имеем соотношение

$$
\begin{aligned}
& \left\|f-\nabla p-P_{k}\right\|_{L^{2}\left(0, T ; L^{2}(\Omega)^{m}\right)} \\
& \quad \leqslant C\left\|A_{1}\left(f-\nabla p-P_{k}\right)\right\|_{L^{2}\left(0, T ; L^{2}(\Omega)^{m}\right)} \rightarrow 0 \text { при } k \rightarrow \infty .
\end{aligned}
$$


Используя введенные выше функции, определим последовательности $v_{\varepsilon}^{s k}$ и $q_{\varepsilon}^{k}$, "приближаюшие" решение задачи (0.4):

$$
v_{\varepsilon}^{s k}=\frac{\varepsilon^{2}}{\nu} a_{\varepsilon} P_{k}+\frac{\varepsilon^{3}}{\nu} w_{\varepsilon}^{s} \nabla P_{k}, \quad q_{\varepsilon}^{k}=p+\varepsilon b_{\varepsilon} P_{k}
$$

Отметим, что, заменяя в $(2.7)$ функции $P_{k}$ и $w_{\varepsilon}^{s}$ на (их пределы при фиксированном $\varepsilon$ и $s, k \rightarrow \infty) f-\nabla p$ и $w_{\varepsilon}$, получим начальные члены асимптотического разложения для решений задачи (0.4). Здесь мы следуем основной идее работы [6]: для доказательства сходимости следует оценить разность точных решений задачи и подходяше сглаженных членов асимптотического разложения и перейти к пределу по параметрам сглаживания и затем по $\varepsilon$. Для осуществления этого плана вычислим предварительно $\operatorname{div} v_{\varepsilon}^{s k}$.

Учитывая обычные для теории осреднения формулы дифференщирования (см., например, [3], [5], [12]), имеем равенства

$$
\begin{aligned}
\operatorname{div} v_{\varepsilon}^{s k} & =\frac{\varepsilon^{2}}{\nu} a_{\varepsilon} \nabla P_{k}+\frac{\varepsilon^{2}}{\nu}\left(\operatorname{div}_{y} w^{s}\right)_{\varepsilon} \nabla P_{k}+\frac{\varepsilon^{3}}{\nu} w_{\varepsilon}^{s} \nabla \nabla P_{k} \\
& =\frac{\varepsilon^{2}}{\nu}\left(\left(\operatorname{div}_{y} w^{s}\right)_{\varepsilon}-A_{1} /|F|+a_{\varepsilon}\right) \nabla P_{k}+\frac{\varepsilon^{3}}{\nu} w_{\varepsilon}^{s} \nabla \nabla P_{k}
\end{aligned}
$$

(в естественных обозначениях, вполне очевидных в координатной записи), где учтено, что $\operatorname{div}_{y} a(y)=0$, и равенство $(2.5)$, умноженное на $\varepsilon^{2} / \nu|F|$. Поскольку функции $w_{\varepsilon}^{s}$ и $P_{k}$ Являются гладкими и финитными, из $(0.4)$ и $(2.8)$ получаем неравенство

$$
\left\|\operatorname{div}\left(u_{\varepsilon}-v_{\varepsilon}^{s k}\right)\right\|_{L^{2}\left(0, T ; L^{2}\left(\Omega_{\varepsilon}\right)\right)} \leqslant \frac{\varepsilon^{2}}{\nu} \delta_{s} C_{k}+\frac{\varepsilon^{3}}{\nu} C_{s k},
$$

где $C_{s k}, C_{k}, \delta_{s}$ не зависят от $\varepsilon$ и, кроме того, $\delta_{s}$ достаточно мало при больших $s$ в соответствии с (2.3). Аналогично, из (0.4) и (2.7) имеем равенство

$$
\begin{gathered}
\left(u_{\varepsilon}-v_{\varepsilon}^{s k}\right)_{t}^{\prime}-\nu \Delta\left(u_{\varepsilon}-v_{\varepsilon}^{s k}\right)+\nabla\left(p_{\varepsilon}-q_{\varepsilon}^{k}\right)=f-\nabla p+\left(\Delta_{y} a-\nabla_{y} b\right)_{\varepsilon} P_{k} \\
-\left(v_{\varepsilon}^{s k}\right)_{t}^{\prime}+2 \varepsilon\left(\nabla_{y} a\right)_{\varepsilon} \nabla P_{k}+\varepsilon^{2} a_{\varepsilon} \Delta P_{k}-\varepsilon b_{\varepsilon} \nabla P_{k} \\
+\varepsilon\left(\Delta_{y} w^{s}\right)_{\varepsilon} \nabla P_{k}+2 \varepsilon^{2}\left(\nabla_{y} w^{s}\right)_{\varepsilon} \nabla \nabla P_{k}+\varepsilon^{3} w_{\varepsilon}^{s} \Delta \nabla P_{k} .
\end{gathered}
$$

Пусть $\varepsilon A_{\varepsilon}^{s k}$ обозначает последние шесть слагаемых в правой части равенства $(2.10)$ и $D_{k}=f-\nabla p-P_{k}, B_{\varepsilon}^{s k}=-a_{\varepsilon}\left(P_{k}\right)_{t}^{\prime}-\varepsilon w_{\varepsilon}^{s}\left(\nabla P_{k}\right)_{t}^{\prime}$. Тогда в силу первого уравнения в (0.8) вьполнено равенство

$$
\left(u_{\varepsilon}-v_{\varepsilon}^{s k}\right)_{t}^{\prime}-\nu \Delta\left(u_{\varepsilon}-v_{\varepsilon}^{s k}\right)+\nabla\left(p_{\varepsilon}-q_{\varepsilon}^{k}\right)=D_{k}+\varepsilon A_{\varepsilon}^{s k}+\left(\varepsilon^{2} / \nu\right) B_{\varepsilon}^{s k} .
$$

Используя (2.1), ограниченность $\Omega$ и гладкость $w_{\varepsilon}^{s}, P_{k}$, имеем неравенства

$$
\begin{gathered}
\left\|A_{\varepsilon}^{s k}\right\|_{L^{2}\left(0, T ; L^{2}\left(\Omega_{\varepsilon}\right)^{m}\right)} \leqslant C_{s k}, \quad\left\|B_{\varepsilon}^{s k}\right\|_{L^{2}\left(0, T ; L^{2}\left(\Omega_{\varepsilon}\right)^{m}\right)} \leqslant C_{s k}, \\
\left\|D_{k}\right\|_{L^{2}\left(0, T ; L^{2}\left(\Omega_{\varepsilon}\right)^{m}\right)} \leqslant \delta_{k},
\end{gathered}
$$

где $C_{s k}, \delta_{k}$ не зависят от $\varepsilon$ и, кроме того, $\delta_{k}$ достаточно мало при больших $k$ в соответствии с (2.6).

Функция $P_{k}$ финитна, и поэтому (см. доказательство леммы 7) найдется $\omega_{k} \subset \subset \Omega$, содержащее $x$-носитель $P_{k}$ для всех $t \in[0, T]$. Поскольку $p_{\varepsilon}$ определено 
с точностью до произвольной функции от $t$, из лемм 2,4 и неравенств $(2.1),(2.4)$ следует, что

$$
\left\|p_{\varepsilon}-q_{\varepsilon}^{k}\right\|_{L^{2}\left(0, T ; L^{2}\left(\omega_{k} \cap \Omega_{\varepsilon}\right)\right)} \leqslant C_{k},
$$

где $C_{k}$ не зависит от $\varepsilon$. Фиксируем $\omega \subset \subset \Omega$. Без ограничения обшности можно считать, что $\omega \subset \omega_{k}$ для всех $k$. В силу ограниченности области $\Omega$ имеем неравенство

$$
\left\|p_{\varepsilon}-q_{\varepsilon}^{k}\right\|_{L^{2}\left(0, T ; L^{1}\left(\omega \cap \Omega_{\varepsilon}\right)\right)} \leqslant C\left\|p_{\varepsilon}-q_{\varepsilon}^{k}\right\|_{L^{2}\left(0, T ; L^{2}\left(\omega_{k} \cap \Omega_{\varepsilon}\right)\right)}
$$

с постоянной $C$, не зависящей от $\varepsilon$ и $k$. Следовательно, для п.в. $t$ определен интеграл $\left|\omega \cap \Omega_{\varepsilon}\right|^{-1} \int_{\omega \cap \Omega_{\varepsilon}}\left(p_{\varepsilon}-q_{\varepsilon}^{k}\right) d x$, вычитая который из $p_{\varepsilon}$, можно считать, что $\int_{\omega \cap \Omega_{\varepsilon}}\left(p_{\varepsilon}-q_{\varepsilon}^{k}\right) d x=0$. При этом равенство (2.11) и неравенство (2.13) сохраняются. У множая скалярно в $L^{2}\left(0, T ; L^{2}\left(\Omega_{\varepsilon}\right)^{m}\right)$ равенство $(2.11)$ на $u_{\varepsilon}-v_{\varepsilon}^{s k}$, как и в лемме 4 получаем

$$
\begin{aligned}
\nu \| \nabla( & \left.u_{\varepsilon}-v_{\varepsilon}^{s k}\right) \|_{L^{2}\left(0, T ; L^{2}\left(\Omega_{\varepsilon}\right)^{m \times m}\right)}^{2} \leqslant \int_{0}^{T}\left(p_{\varepsilon}-q_{\varepsilon}^{k}, \operatorname{div}\left(u_{\varepsilon}-v_{\varepsilon}^{s k}\right)\right)_{L^{2}\left(\omega_{k} \cap \Omega_{\varepsilon}\right)} d t \\
& +\int_{0}^{T}\left(D_{k}+\varepsilon A_{\varepsilon}^{s k}+\frac{\varepsilon^{2}}{\nu} B_{\varepsilon}^{s k},\left(u_{\varepsilon}-v_{\varepsilon}^{s k}\right)\right)_{L^{2}\left(\Omega_{\varepsilon}\right)^{m}} d t \\
\leqslant & \left(\frac{\varepsilon^{2}}{\nu} \delta_{s} C_{k}+\frac{\varepsilon^{3}}{\nu} C_{s k}\right)\left\|p_{\varepsilon}-q_{\varepsilon}^{k}\right\|_{L^{2}\left(0, T ; L^{2}\left(\omega_{k} \cap \Omega_{\varepsilon}\right)\right)} \\
& +\left(\varepsilon \delta_{k}+\varepsilon^{2} C_{s k}+\frac{\varepsilon^{3}}{\nu} C_{s k}\right)\left\|\nabla\left(u_{\varepsilon}-v_{\varepsilon}^{s k}\right)\right\|_{L^{2}\left(0, T ; L^{2}\left(\Omega_{\varepsilon}\right)^{m \times m}\right)} \\
\leqslant & \frac{\varepsilon^{2}}{\nu} \delta_{s} C_{k}+\frac{\varepsilon^{3}}{\nu} C_{s k}+\frac{\varepsilon^{2} \delta_{k}^{2}}{2 \nu}+\frac{\varepsilon^{4}}{2 \nu} C_{s k}^{2}+\frac{\varepsilon^{6}}{2 \nu^{3}} C_{s k}^{2} \\
& +\frac{\nu}{2}\left\|\nabla\left(u_{\varepsilon}-v_{\varepsilon}^{s k}\right)\right\|_{L^{2}\left(0, T ; L^{2}\left(\Omega_{\varepsilon}\right)^{m \times m}\right)}^{2}
\end{aligned}
$$

где учтены неравенства $(2.9),(2.12),(2.13)$, лемма 1 и принадлежность $x$-носителя функции $\operatorname{div}\left(u_{\varepsilon}-v_{\varepsilon}^{s k}\right)=-\operatorname{div} v_{\varepsilon}^{s k}$ множеству $\omega_{k} \cap \Omega_{\varepsilon}$. Здесь использовано также $\varepsilon$-неравенство, записанное в виде $|\alpha \beta| \leqslant(\varepsilon / 2) \alpha^{2}+\beta^{2} /(2 \varepsilon)$ для положительных $\varepsilon$ и произвольных $\alpha, \beta$. Вновь применяя лемму 1 , из (2.14) имеем

$$
\begin{aligned}
& \left(\nu / \varepsilon^{2}\right)\left\|u_{\varepsilon}-v_{\varepsilon}^{s k}\right\|_{L^{2}\left(0, T ; L^{2}(\Omega)^{m}\right)} \leqslant(\nu / \varepsilon) C\left\|\nabla\left(u_{\varepsilon}-v_{\varepsilon}^{s k}\right)\right\|_{L^{2}\left(0, T ; L^{2}\left(\Omega_{\varepsilon}\right)^{m \times m}\right)} \\
& \quad \leqslant \sqrt{\varepsilon} C_{s k}+\delta_{s} C_{k}+\delta_{k} C+\left(\varepsilon^{2} / \nu\right) C_{s k} .
\end{aligned}
$$

Здесь и далее $C_{s k}, C_{k}, \delta_{s}, \delta_{k}$ не зависят от $\varepsilon$ и, кроме того, $\delta_{s}, \delta_{k}$ достаточно малы при больших $s$ и $k$.

Аналогично, умножая скалярно в $L^{2}\left(0, T ; L^{2}\left(\Omega_{\varepsilon}\right)^{m}\right)$ равенство (2.11) на $\left(u_{\varepsilon}-v_{\varepsilon}^{s k}\right)_{t}^{\prime}$, получаем оценку

$$
\begin{aligned}
\|\left(u_{\varepsilon}-\right. & \left.v_{\varepsilon}^{s k}\right)_{t}^{\prime} \|_{L^{2}\left(0, T ; L^{2}(\Omega)^{m}\right)}^{2} \leqslant \int_{0}^{T}\left(q_{\varepsilon}^{k}-p_{\varepsilon}, \operatorname{div}\left(v_{\varepsilon}^{s k}\right)_{t}^{\prime}\right)_{L^{2}\left(\omega_{k} \cap \Omega_{\varepsilon}\right)} d t \\
& +\int_{0}^{T}\left(D_{k}+\varepsilon A_{\varepsilon}^{s k}+\left(\varepsilon^{2} / \nu\right) B_{\varepsilon}^{s k},\left(u_{\varepsilon}-v_{\varepsilon}^{s k}\right)_{t}^{\prime}\right)_{L^{2}\left(\Omega_{\varepsilon}\right)^{m}} d t
\end{aligned}
$$

из которой и из $(2.8),(2.12)$ следует неравенство

$$
\left\|\left(u_{\varepsilon}-v_{\varepsilon}^{s k}\right)_{t}^{\prime}\right\|_{L^{2}\left(0, T ; L^{2}(\Omega)^{m}\right)} \leqslant \sqrt{\varepsilon} C_{s k}+\delta_{s} C_{k}+\delta_{k} C+\left(\varepsilon^{2} / \nu\right) C_{s k} .
$$


Следовательно, используя леммы 2,3 и равенство (2.11), имеем неравенства

$$
\begin{aligned}
\| p_{\varepsilon}- & q_{\varepsilon}^{k}\left\|_{L^{2}\left(0, T ; L^{2}\left(\omega \cap \Omega_{\varepsilon}\right)\right)} \leqslant(C / \varepsilon)\right\| \nabla\left(p_{\varepsilon}-q_{\varepsilon}^{k}\right) \|_{L^{2}\left(0, T ; H^{-1}\left(\Omega_{\varepsilon}\right)^{m}\right)} \\
\leqslant & C\left\|D_{k}+\varepsilon A_{\varepsilon}^{s k}+\left(\varepsilon^{2} / \nu\right) B_{\varepsilon}^{s k}-\left(u_{\varepsilon}-v_{\varepsilon}^{s k}\right)_{t}^{\prime}\right\|_{L^{2}\left(0, T ; L^{2}\left(\Omega_{\varepsilon}\right)^{m}\right)} \\
& +(\nu / \varepsilon) C\left\|\nabla\left(u_{\varepsilon}-v_{\varepsilon}^{s k}\right)\right\|_{L^{2}\left(0, T ; L^{2}\left(\Omega_{\varepsilon}\right)^{m \times m}\right)} \\
\leqslant & \sqrt{\varepsilon} C_{s k}+\delta_{s} C_{k}+\delta_{k} C+\left(\varepsilon^{2} / \nu\right) C_{s k},
\end{aligned}
$$

где учтены неравенства $(2.12),(2.15)$ и $(2.16)$.

Из (2.1), (2.3), (2.6) и (2.17) уже следует соотношение (0.7) теоремы 1 . Действительно, для произвольного $\delta>0$ можно выбрать $k$ и $s$ так, чтобы $\delta_{s} C_{k}+\delta_{k} C<\delta / 2$. Тогда, учитывая $(0.1)$ и $(2.1)$, найдется такое $\varepsilon(\delta)$, что $\varepsilon\left\|b_{\varepsilon} P_{k}\right\|_{L^{2}\left(0, T ; L^{2}\left(\omega \cap \Omega_{\varepsilon}\right)\right)}+$ $\sqrt{\varepsilon} C_{s k}+\left(\varepsilon^{2} / \nu\right) C_{s k}<\delta / 2$ и

$$
\left\|p_{\varepsilon}-p\right\|_{L^{2}\left(0, T ; L^{2}\left(\omega \cap \Omega_{\varepsilon}\right)\right)}<\delta \quad \text { при } \quad \varepsilon<\varepsilon(\delta),
$$

что эквивалентно соотношению $(0.7)$, поскольку $p_{\varepsilon}$ и $p$ определены с точностью до произвольных функций от $t$.

Для завершения доказательства теоремы 1 достаточно доказать (см., например, [3]), что для фиксированного $\varphi \in C_{0}^{\infty}((0, T) \times \Omega)^{m}$ и произвольного $\delta>0$ найдется такое $\varepsilon(\delta)$, что

$$
I=\left|\int_{0}^{T}\left(\frac{\nu}{\varepsilon^{2}} u_{\varepsilon}-A_{1}(f-\nabla p), \varphi\right)_{L^{2}(\Omega)^{m}} d t\right|<\delta \quad \text { при } \quad \varepsilon<\varepsilon(\delta) .
$$

Из соотношений $(2.2),(2.6),(2.7)$ и (2.15) имеем неравенства

$$
\begin{aligned}
I \leqslant & \left|\int_{0}^{T}\left(\frac{\nu}{\varepsilon^{2}} u_{\varepsilon}-a_{\varepsilon} P_{k}, \varphi\right)_{L^{2}(\Omega)^{m}} d t\right|+\left|\int_{0}^{T}\left(\left(a_{\varepsilon}-A_{1}\right) P_{k}, \varphi\right)_{L^{2}(\Omega)^{m}} d t\right| \\
& +\left|\int_{0}^{T}\left(A_{1}\left(P_{k}-f+\nabla p\right), \varphi\right)_{L^{2}(\Omega)^{m}} d t\right| \\
\leqslant & C\left\|\frac{\nu}{\varepsilon^{2}} u_{\varepsilon}-a_{\varepsilon} P_{k}\right\|_{L^{2}\left(0, T ; L^{2}(\Omega)^{m}\right)}+\varepsilon\left|\int_{0}^{T}\left(W_{\varepsilon}, \nabla\left(P_{k} \varphi\right)\right)_{L^{2}(\Omega)^{m \times m \times m}} d t\right| \\
& +C\left\|A_{1}\left(P_{k}-f+\nabla p\right)\right\|_{L^{2}\left(0, T ; L^{2}(\Omega)^{m}\right)} \\
\leqslant & \sqrt{\varepsilon} C_{s k}+\delta_{s} C_{k}+\delta_{k} C+\left(\varepsilon^{2} / \nu\right) C_{s k} .
\end{aligned}
$$

Таким образом, найдется $\varepsilon(\delta)$, удовлетворяющее $(2.18)$, и теорема 1 доказана.

Отметим, что из (2.15) еще не следует соотношение

$$
\left\|\frac{\nu}{\varepsilon^{2}} u_{\varepsilon}-a_{\varepsilon}(f-\nabla p)\right\|_{L^{2}\left(0, T ; L^{2}(\Omega)^{m}\right)} \rightarrow 0 \quad \text { при } \quad \varepsilon \rightarrow 0 .
$$

Однако, например, для $m=3$ и $\partial F_{1} \in C^{2}$ это соотношение имеет место. Действительно, в этом случае $a(y) \in W_{2}^{2}(F) \subset C^{0}(\bar{F})$ (см., например, [1]), и поэтому $\max _{\bar{\Omega}}\left|a_{\varepsilon}\right| \leqslant C$ с постоянной $C$, не зависящей от $\varepsilon$. Таким образом,

$$
\begin{aligned}
\| \frac{\nu}{\varepsilon^{2}} u_{\varepsilon} & -a_{\varepsilon}(f-\nabla p)\left\|_{L^{2}\left(0, T ; L^{2}(\Omega)^{m}\right)}+\right\| a_{\varepsilon}\left(P_{k}-f+\nabla p\right) \|_{L^{2}\left(0, T ; L^{2}(\Omega)^{m}\right)} \\
& \leqslant\left\|\frac{\nu}{\varepsilon^{2}} u_{\varepsilon}-a_{\varepsilon} P_{k}\right\|_{L^{2}\left(0, T ; L^{2}(\Omega)^{m}\right)}+C\left\|P_{k}-f+\nabla p\right\|_{L^{2}\left(0, T ; L^{2}(\Omega)^{m}\right)}, \\
& \leqslant\left\|\frac{\nu}{\varepsilon^{2}} u_{\varepsilon}-a_{\varepsilon} P_{k}\right\|_{L^{2}\left(0, T ; L^{2}(\Omega)^{m}\right)}+C
\end{aligned}
$$

и $(2.20)$ следует из $(2.6),(2.7)$ и $(2.15)$. 
ДоКАЗАТЕЛЬСТВО ТЕОРЕМЫ 2. В соответствии с результатами [1], [2] решение $B(y)$ задачи $(0.10)$ с компонентами из $H_{P}^{1}(F) \cap L^{2}(F) / \mathbb{R}$ существует и единственно. Здесь $H_{P}^{1}(F)$ - соболевское пространство 1-периодических функций из $H^{1}(F)$ (см., например, [5], [12]). Аналогично, решение $a(y, t), b(y, t)$ задачи $(0.9)$ с компонентами из $L^{2}\left(0, \infty ; H_{P}^{1}(F, S)\right), L^{2}\left(0, \infty ; L^{2}(F) / \mathbb{R}\right)$ существует и единственно. Обозначим через $\lambda_{l}$ и $e_{l}$ собственные значения и собственные функции 1 -периодической задачи Стокса на $F$ :

$$
-\Delta e_{l}+\nabla b_{l}=\lambda_{l} e_{l}, \quad \operatorname{div} e_{l}=0 \quad \text { в } F, \quad e_{l}=0 \text { на } S .
$$

Для каждого $l=1,2, \ldots$ рассмотрим матричнозначную функцию $a_{l}(y)$, столбцами которой являются $e_{l}(y)$. В соответствии с [2] имеем равенства

$$
a(y, t)=\sum_{l=1}^{\infty}\left\langle Q(E) a_{l}\right\rangle a_{l}^{*}(y) e^{-\lambda_{l} t}=\sum_{l=1}^{\infty}\left\langle a_{l}\right\rangle a_{l}^{*}(y) e^{-\lambda_{l} t},
$$

где * обозначает матричное сопряжение и ряды сходятся в пространстве $L^{2}\left(0, \infty ; H_{P}^{1}(F)^{m \times m}\right)$. Кроме того [2], ряд

$$
\sum_{l=1}^{\infty}\left\langle a_{l}\right\rangle\left\langle a_{l}\right\rangle^{*} e^{-\lambda_{l} t}=\langle a(y, t)\rangle=A_{2}(t)
$$

сходится в $\mathbb{R}^{m \times m}$ для каждого неотрицательного $t$, поскольку $Q(E) \in H(F)$ и все $\lambda_{l}$ положительны. Из последнего утверждения следует, что

$$
A_{2}(t)_{t}^{\prime}=\left\langle a(y, t)_{t}^{\prime}\right\rangle \in L^{1}\left(0, \infty ; \mathbb{R}^{m \times m}\right) .
$$

Действительно, прямое вычисление дает неравенство

$$
\begin{aligned}
\left\|\left\langle a(y, t)_{t}^{\prime}\right\rangle\right\|_{L^{1}\left(0, \infty ; \mathbb{R}^{m \times m}\right)} & =\int_{0}^{\infty}\left\|\sum_{l=1}^{\infty}\left\langle a_{l}\right\rangle\left\langle a_{l}\right\rangle^{*}\left(-\lambda_{l}\right) e^{-\lambda_{l} t}\right\|_{\mathbb{R}^{m \times m}} d t \\
& \leqslant \sum_{l=1}^{\infty}\left\|\left\langle a_{l}\right\rangle\right\|_{\mathbb{R}^{m \times m}}^{2} \int_{0}^{\infty} \lambda_{l} e^{-\lambda_{l} t} d t=\sum_{l=1}^{\infty}\left\|\left\langle a_{l}\right\rangle\right\|_{\mathbb{R}^{m \times m}}^{2}
\end{aligned}
$$

и сходимость последнего ряда эквивалентна сходимости при $t=0$ ряда (2.22). Здесь и в дальнейшем $\|\cdot\|_{\mathbb{R}^{m} \times m}$ обозначает операторную норму матрищы.

Определим $a_{\varepsilon}=a(x / \varepsilon, t),\left(\nabla_{y} a\right)_{\varepsilon}=\left(\nabla_{y} a\right)(x / \varepsilon, t), b_{\varepsilon}=b(x / \varepsilon, t), B_{\varepsilon}=B(x / \varepsilon)$ и продолжим эти функции по $\varepsilon$-периодичности на $\Omega_{\varepsilon}$ и $a_{\varepsilon}$ нулем на $\Omega \backslash \Omega_{\varepsilon}$. Из ограниченности области $\Omega_{\varepsilon}$ и аналога леммы 4 для задачи $(0.9)$ следуют неравенства

$$
\begin{array}{rlrl}
\left\|a_{\varepsilon}\right\|_{L^{2}\left(0, \infty ; L^{2}(\Omega)^{m \times m}\right)} & \leqslant C, & \left\|b_{\varepsilon}\right\|_{L^{2}\left(0, \infty ; L^{2}\left(\Omega_{\varepsilon}\right)^{m}\right)} & \leqslant C, \\
\left\|\left(\nabla_{y} a\right)_{\varepsilon}\right\|_{L^{2}\left(0, \infty ; L^{2}\left(\Omega_{\varepsilon}\right)^{m \times m \times m}\right)} & \leqslant C, & \left\|B_{\varepsilon}\right\|_{L^{2}\left(\Omega_{\varepsilon}\right)^{m}} \leqslant C .
\end{array}
$$

Кроме того [10], найдется такое $W_{\varepsilon}(x, t) \in L^{2}\left(0, \infty ; H^{1}(\Omega)^{m \times m \times m}\right)$, что $\left\|W_{\varepsilon}\right\|_{L^{2}\left(0, \infty ; L^{2}(\Omega)^{m \times m \times m}\right)} \leqslant C$ и

$$
a_{\varepsilon}-A_{2}=\varepsilon \operatorname{div}\left(W_{\varepsilon}\right)
$$


Для доказательства этого равенства достаточно повторить для п.в. $t$ доказательство равенства $(2.2)$ и учесть принадлежность $a_{\varepsilon}$ и $A_{2}$ соответствуюшим пространствам.

Аналогично, поскольку $A_{2} /|F|-a \in L^{2}\left(0, \infty ;\left(L^{2}(F) / \mathbb{R}\right)^{m \times m}\right)$, найдется такое $w(y, t) \in L^{2}\left(0, \infty ; H_{P}^{1}(F, S)^{m \times m \times m}\right)$, что $\operatorname{div}_{y} w=A_{2} /|F|-a$. Для доказательства этого равенства можно также воспользоваться представлениями $(2.21),(2.22)$ и разложить $w(y, t)$ в ряд по экспонентам, коэффициенты которого находятся с помощью леммы 8 . Тогда сходимость этого ряда в пространстве $L^{2}\left(0, \infty ; H_{P}^{1}(F, S)^{m \times m \times m}\right)$ будет следовать из сходимости рядов $(2.21),(2.22)$ в соответствующих пространствах. По определению найдется последовательность $\left\{w^{s}(y, t)\right\} \subset L^{2}\left(0, \infty ; C_{P}^{\infty}(F, S)^{m \times m \times m}\right)$, сходящаяся к $w(y, t)$ в норме $L^{2}\left(0, \infty ; H_{P}^{1}(F, S)^{m \times m \times m}\right)$. Определим $w_{\varepsilon}^{s}=w^{s}(x / \varepsilon, t), \quad\left(\operatorname{div}_{y} w^{s}\right)_{\varepsilon}=$ $\left(\operatorname{div}_{y} w^{s}\right)(x / \varepsilon, t)$, продолжим эти функции по $\varepsilon$-периодичности на $\Omega_{\varepsilon}$ и $w_{\varepsilon}$ нулем на $\Omega \backslash \Omega_{\varepsilon}$. Тогда равномерно по $\varepsilon$ вьполнено соотношение

$$
\begin{aligned}
& \left\|\left(\operatorname{div}_{y} w^{s}\right)_{\varepsilon}-A_{2} /|F|+a_{\varepsilon}\right\|_{L^{2}\left(0, \infty ; L^{2}\left(\Omega_{\varepsilon}\right)^{m \times m}\right)} \\
& \quad \leqslant C\left\|\operatorname{div}_{y} w^{s}(y)-A_{2} /|F|+a(y)\right\|_{L^{2}\left(0, \infty ; L^{2}(F)^{m \times m}\right)} \rightarrow 0 \quad \text { при } \quad s \rightarrow \infty .
\end{aligned}
$$

Осредненная задача для "предельного" давления $p$ в рассматриваем случае имеет вид (0.12), или, в вариационной форме,

$$
\int_{0}^{\infty}\left(A_{2} *(f-\nabla p), \nabla \varphi\right)_{L^{2}(\Omega)^{m}} d t=0 \quad \forall \varphi \in L^{2}\left(0, \infty ; H^{1}(\Omega) \cap L^{2}(\Omega) / \mathbb{R}\right) .
$$

Здесь предполагается, что $f \in L^{2}\left(0, \infty ; L^{2}(\Omega)^{m}\right)$. Докажем, что существует решение $p \in L^{2}\left(0, \infty ; H^{1}(\Omega) \cap L^{2}(\Omega) / \mathbb{R}\right)$ этой задачи, удовлетворяющее неравенству (2.4). В соответствии с [16] для этого достаточно доказать, что для каждого $\sigma \in C^{+}$сушествует решение задачи (образа задачи (2.27) при преобразовании Лапласа):

$$
(\hat{A}(\sigma)(\hat{f}(\sigma)-\nabla \hat{p}(\sigma)), \nabla \varphi)_{L^{2}(\Omega)^{m}}=0 \quad \forall \varphi \in H^{1}(\Omega) \cap L^{2}(\Omega) / \mathbb{R},
$$

для которого выполнено неравенство

$$
\|\hat{p}(\sigma)\|_{L^{2}(\Omega)}+\|\nabla \hat{p}(\sigma)\|_{L^{2}(\Omega)^{m}} \leqslant C\|\hat{f}(\sigma)\|_{L^{2}(\Omega)^{m}}
$$

с постоянной $C$, не зависяшей от $\sigma \in C^{+}$. Действительно, тогда $\hat{p}(\sigma)$ голоморфно, $\|\nabla \hat{p}\|_{E_{0}\left(C^{+} ; L^{2}(\Omega)^{m}\right)} \leqslant C\|\hat{f}\|_{E_{0}\left(C^{+} ; L^{2}(\Omega)^{m}\right)}$ и сушествование подходящего $p$ будет следовать из теоремы 9 и неравенства Пуанкаре. Здесь $\hat{A}, \hat{f}$ и $\hat{p}$ обозначают преобразования Лапласа от $A_{2}, f$ и $p$.

Применяя преобразование Лапласа к первому уравнению в (0.9), получаем

$$
\sigma \hat{a}-\Delta \hat{a}+\nabla \hat{b}=E-\nabla B .
$$

Будем обозначать через $\hat{a}^{*}$ матрицу, транспонированную и комплексно-сопряженную к $\hat{a}$. Умножая равенство (2.30) на $\hat{a}^{*}$ справа, сопряженное к (2.30) равенство на $\hat{a}$ слева и интегрируя полученные соотношения по $F$, имеем

$$
\begin{aligned}
& \sigma\left\langle\hat{a} \hat{a}^{*}\right\rangle+\left\langle\nabla \hat{a} \nabla \hat{a}^{*}\right\rangle=\hat{A}^{*}, \\
& \bar{\sigma}\left\langle\hat{a} \hat{a}^{*}\right\rangle+\left\langle\nabla \hat{a} \nabla \hat{a}^{*}\right\rangle=\hat{A}
\end{aligned}
$$


для каждого $\sigma \in C^{+}$и по непрерывности [16] для каждого $\sigma \in \bar{C}^{+}=\{\sigma \in C: \sigma=$ $\alpha+i \beta, \alpha \geqslant 0\}$. Пусть $\sigma=\alpha+i \beta$; тогда

$$
\operatorname{Im} \hat{A}=-\beta\left\langle\hat{a} \hat{a}^{*}\right\rangle, \quad \operatorname{Re} \hat{A}=\alpha\left\langle\hat{a} \hat{a}^{*}\right\rangle+\left\langle\nabla \hat{a} \nabla \hat{a}^{*}\right\rangle
$$

являются симметричными матрицами. Матрицы $\left\langle\hat{a} \hat{a}^{*}\right\rangle,\left\langle\nabla \hat{a} \nabla \hat{a}^{*}\right\rangle$ и поэтому $\operatorname{Re} \hat{A}$ неотрицательно определены. Действительно, для каждого $\sigma \in \bar{C}^{+}$и $\xi \in \mathbb{R}^{m}$ из $(2.32)$ получаем

$$
\xi(\operatorname{Re} \hat{A}) \xi^{*}=\alpha\|\xi \hat{a}\|_{L^{2}(F)^{m}}^{2}+\|\nabla(\xi \hat{a})\|_{L^{2}(F)^{m \times m}}^{2} \geqslant 0 .
$$

Фиксируем $\sigma \in \bar{C}^{+}$. В соответствии с [3] существует такая соленоидальная матрица $е$ с компонентами из $H_{P}^{1}(F, S)$, что $\langle e\rangle=E$ и для $\xi \in \mathbb{R}^{m}$ из (2.30) следует, что

$$
\sigma\left\langle\xi \hat{a} e \xi^{*}\right\rangle+\left\langle\nabla(\xi \hat{a}) \nabla\left(e \xi^{*}\right)\right\rangle=\xi^{2} .
$$

Предположим, что $\xi(\operatorname{Re} \hat{A}) \xi^{*}=0$ для некоторого $\xi \in \mathbb{R}^{m}$. Тогда, учитывая $(2.33)$, в $L^{2}(F)^{m}$ и $L^{2}(F)^{m \times m}$ выполнены равенства $\xi \hat{a}=0, \nabla(\xi \hat{a})=0$, и поэтому $\xi=0$ в силу $(2.34)$.

Таким образом, для каждого $\sigma \in \bar{C}^{+}$матрица $\operatorname{Re} \hat{A}$ положительно определена и найдется такое положительное $C(\sigma)$, что

$$
C(\sigma) \xi^{2} \leqslant \xi(\operatorname{Re} \hat{A}) \xi^{*} \quad \text { для } \quad \forall \xi \in \mathbb{R}^{m} .
$$

Обозначим через $K$ некоторое компактное подмножество из $\bar{C}^{+}$. Тогда в силу компактности единичной сферы в $\mathbb{R}^{m}$ для $\sigma \in K$ постоянную $C(\sigma)$ в $(2.35)$ можно выбрать не зависящей от $\sigma$. Поскольку $a \in L^{2}\left(0, \infty ; L^{2}(F)^{m \times m}\right)$, из представления (2.22) следует, что $A_{2} \in L^{1}\left(0, \infty ; \mathbb{R}^{m \times m}\right)$, и поэтому

$$
\|\hat{A}(\sigma)\|_{C^{m \times m}}=\left\|\int_{0}^{\infty} e^{-\sigma t} A_{2}(t) d t\right\|_{C^{m \times m}} \leqslant \int_{0}^{\infty}\left\|A_{2}(t)\right\|_{C^{m \times m}} d t \leqslant C,
$$

где $C$ не зависит от $\sigma \in \bar{C}^{+}$. Из неравенств (2.35) и (2.36) следует (см., например, $[17$, гл. $3, \S 1])$, что для каждого $\sigma \in \bar{C}^{+}$решение задачи $(2.28)$, принадлежащее $H^{1}(\Omega) \cap L^{2}(\Omega) / \mathbb{R}$, существует и единственно. Однако из неравенства $(2.35)$ выводится неравенство (2.29) только для $\sigma \in K$.

Следуя, например, [18], проинтегрируем по частям в преобразовании Лаплаca $\hat{A}(\sigma)$ от $A_{2}(t)$. Тогда

$$
\begin{aligned}
\hat{A}(\sigma) & =\int_{0}^{\infty} e^{-\sigma t}\langle a\rangle d t=\left.\sigma^{-1}\langle a\rangle\right|_{t=0}+\sigma^{-1} \int_{0}^{\infty} e^{-\sigma t}\langle a\rangle_{t}^{\prime} d t \\
& =\sigma^{-1}\langle Q(E)\rangle+\sigma^{-1} \int_{0}^{\infty} e^{-\sigma t} A_{2}(t)_{t}^{\prime} d t .
\end{aligned}
$$

Эти равенства имеют смысл в силу соотношений (2.22) и (2.23). Более того, последний интеграл стремится к нулевой матрище при $|\sigma| \rightarrow \infty$ в силу $(2.23)$ и леммы Римана-Лебега (см., например, [18]). Таким образом, $\sigma \hat{A}(\sigma)=\langle Q(E)\rangle+I$, где 
$\|I\|_{C} \times m$ стремится к нулю при $|\sigma| \rightarrow \infty$. Известно [12], что симметрическая матрица $\langle Q(E)\rangle$ с постоянными действительными компонентами положительно определена, и поэтому для достаточно больших $\sigma_{0}$ при $|\sigma| \geqslant \sigma_{0}$ выполнены неравенства

$$
C_{1} \xi^{2} \leqslant \xi \operatorname{Re}(\sigma \hat{A}) \xi^{*}, \quad|\sigma|\|\hat{A}(\sigma)\|_{C^{m \times m}} \leqslant C_{2}
$$

с постоянными $C_{1}$ и $C_{2}$, не зависящими от $\xi \in \mathbb{R}^{m}$ и от $\sigma$ при $|\sigma| \geqslant \sigma_{0}$. Следовательно, умножая (2.28) на $\sigma$ и учитывая (2.37), получаем неравенство (2.29) для $|\sigma| \geqslant \sigma_{0}$. Поскольку множество $\left\{\sigma \in \bar{C}^{+}:|\sigma| \leqslant \sigma_{0}\right\}$ компактно, неравенство (2.29) выполнено для всех $\sigma \in \bar{C}^{+}$, что завершает доказательство сушествования подходящего решения задачи (2.27).

Из неравенств $(2.35),(2.37)$ следует, что матрица $\hat{A}(\sigma)^{-1}$ определена для всех $\sigma \in \bar{C}^{+}$, голоморфна и удовлетворяет неравенству

$$
\left\|\hat{A}(\sigma)^{-1}\right\|_{C^{m \times m}} \leqslant C(1+|\sigma|)
$$

с постоянной $C$, не зависящей от $\sigma \in \bar{C}^{+}$. Следовательно [19, гл. $\left.2, \S 10\right]$, существует матричнозначное распределение $A_{2}^{-1}(t)$ с компонентами из $D_{+}^{\prime}(0)$, преобразование Лапласа которого совпадает с $\hat{A}(\sigma)^{-1}$. Кроме того, оператор $A_{2}^{-1}(t) *$ определен на $C_{0}^{\infty}(0, \infty)^{m}$ и переводит это пространство в пространство $S(0, \infty)^{m}$ гладких, быстро убывающих на бесконечности и обнуляющихся в нуле вектор-функций.

Пусть $G=A_{2} *(f-\nabla p)$. Тогда $\operatorname{div} G=0$ в $\Omega$ и $(G, n)=0$ на $\partial \Omega$. В силу леммы 7 найдется последовательность $\left\{G_{k}\right\} \subset C_{0}^{\infty}((0, T) \times \Omega)^{m}$ соленоидальных вектор-функций, сходящаяся к $G$ в норме $L^{2}\left(0, \infty ; L^{2}(\Omega)^{m}\right)$. Для каждого $k=1,2, \ldots$ вектор-функция $G_{k}$ является конечной линейной комбинацией векторов вида $a_{s}(t) \varphi_{l}(x)$ с $a_{s}(t) \in C_{0}^{\infty}(0, \infty)$. Следовательно, вектор-функция $P_{k}=A_{2}^{-1}(t) * G_{k}$ определена на $(0, \infty) \times \Omega$, финитна по $x$, быстро убывает по $t$ и является гладкой по $t$ и $x$. Кроме того, $A_{2} * P_{k}=G_{k}$, и поэтому

$$
\operatorname{div} A_{2} * P_{k}=A_{2} * \nabla P_{k}=0 .
$$

Пусть $R \in L^{1}(0, \infty)$ и $g \in L^{2}\left(0, \infty ; L^{2}(\Omega)\right)$. Тогда $R * g=g * R$, и, повторяя доказательство соответствуюшего неравенства из $[20, \S 3.1 .6]$, заключаем, что

$$
\|R * g\|_{L^{2}\left(0, \infty ; L^{2}(\Omega)\right)} \leqslant\|R\|_{L^{1}(0, \infty)}\|g\|_{L^{2}\left(0, \infty ; L^{2}(\Omega)\right)} .
$$

Кроме того, для подходяших $R$ и $g$ имеем равенства

$$
(R * g)_{t}^{\prime}=R(0) g+R_{t}^{\prime} * g=R g(0)+R * g_{t}^{\prime} .
$$

Предположим дополнительно в лемме 7 , что $u \in H_{0}^{1}(0, \infty ; H(\Omega))$. Тогда последовательность $\left\{u_{k}\right\} \subset C_{0}^{\infty}((0, \infty) \times \Omega)^{m}$, определенная в этой лемме, сходится к $u$ в норме $H_{0}^{1}\left(0, \infty ; L^{2}(\Omega)^{m}\right)$. Действительно, по определению множество $C_{0}^{\infty}(0, \infty)$ плотно в $H_{0}^{1}(0, \infty)$. Поэтому можно считать, что последовательность $\left\{a_{s}\right\} \subset C_{0}^{\infty}(0, \infty)$, использованная при доказательстве леммы 7 , образует ортонормированный базис в $H_{0}^{1}(0, \infty)$. Следовательно, конечные линейные комбинации векторов вида $a_{s} \varphi_{l}$ образуют плотное подмножество в $H_{0}^{1}(0, \infty ; H(\Omega))$.

Непосредственно из определений и (2.41) имеем равенства $G(0)=0$ и $G_{t}^{\prime}=$ $\langle Q(E)\rangle(f-\nabla p)+A_{2}(t)_{t}^{\prime} *(f-\nabla p)$. Учитывая соотношения $(2.23),(2.27)$ и $(2.40)$, 
заключаем, что $G \in H_{0}^{1}(0, \infty ; H(\Omega))$. Следовательно, $\left\{G_{k}\right\} \subset C_{0}^{\infty}((0, \infty) \times \Omega)^{m}$ сходится к $G$ в норме $H_{0}^{1}\left(0, \infty ; L^{2}(\Omega)^{m}\right)$. Тогда из теоремы 9 следует, что

$$
\left\|\hat{A}\left(f-\nabla \hat{p}-\widehat{P}_{k}\right)\right\|_{E_{0}\left(C^{+} ; L^{2}(\Omega)^{m}\right)}+\left\|\sigma \hat{A}\left(f-\nabla \hat{p}-\widehat{P}_{k}\right)\right\|_{E_{0}\left(C^{+} ; L^{2}(\Omega)^{m}\right)} \rightarrow 0
$$

при $k \rightarrow \infty$, и поэтому, используя неравенства $(2.35)$ и $(2.37)$, имеем соотношение

$$
\left\|f-\nabla p-P_{k}\right\|_{L^{2}\left(0, \infty ; L^{2}(\Omega)^{m}\right)} \rightarrow 0 \quad \text { при } \quad k \rightarrow \infty .
$$

Пусть $R \in L^{2}\left(0, \infty ; L^{2}\left(\Omega_{\varepsilon}\right)\right)$ и $g=a \varphi$ с $a \in L^{1}(0, \infty)$ и $\varphi \in C_{0}^{\infty}(\Omega)^{m}$. Тогда свертка $R * g$ определена на $(0, \infty) \times \Omega_{\varepsilon}$ и выполнено неравенство

$$
\|R * g\|_{L^{2}\left(0, \infty ; L^{2}\left(\Omega_{\varepsilon}\right)\right)} \leqslant\|a\|_{L^{1}(0, \infty)}\|\varphi\|_{C^{0}(\bar{\Omega})^{m}}\|R\|_{L^{2}\left(0, \infty ; L^{2}\left(\Omega_{\varepsilon}\right)^{m \times m}\right)} .
$$

Действительно, используя для оценки каждой компоненты $R * g=a * R \varphi$ неравенство (2.40), получаем $\|R * g\|_{L^{2}\left(0, \infty ; L^{2}\left(\Omega_{\varepsilon}\right)\right)} \leqslant\|a\|_{L^{1}(0, \infty)}\|R \varphi\|_{L^{2}\left(0, \infty ; L^{2}\left(\Omega_{\varepsilon}\right)\right)}$, что и доказывает (2.43). Таким образом, определены последовательности $v_{\varepsilon}^{s k}$ и $q_{\varepsilon}^{k}$, "приближаюшие" решение задачи (0.4):

$$
v_{\varepsilon}^{s k}=\frac{\varepsilon^{2}}{\nu} a_{\varepsilon} * P_{k}+\frac{\varepsilon^{3}}{\nu} w_{\varepsilon}^{s} * \nabla P_{k}, \quad q_{\varepsilon}^{k}=p+\varepsilon b_{\varepsilon} * P_{k}+\varepsilon B_{\varepsilon} P_{k} .
$$

Учитьвая (2.39) и соленоидальность $a(y, t)$, получаем равенство

$$
\operatorname{div} v_{\varepsilon}^{s k}=\frac{\varepsilon^{2}}{\nu}\left(\left(\operatorname{div}_{y} w^{s}\right)_{\varepsilon}-A_{2} /|F|+a_{\varepsilon}\right) * \nabla P_{k}+\frac{\varepsilon^{3}}{\nu} w_{\varepsilon}^{s} * \nabla \nabla P_{k}
$$

Следовательно, в силу (2.26) и (2.43) выполнено неравенство (2.9). Аналогично, из $(0.4),(2.41)$ и $(2.44)$ имеем равенство

$$
\begin{aligned}
\left(u_{\varepsilon}-v_{\varepsilon}^{s k}\right)_{t}^{\prime}-\nu \Delta\left(u_{\varepsilon}-v_{\varepsilon}^{s k}\right)+\nabla\left(p_{\varepsilon}-q_{\varepsilon}^{k}\right) & \\
= & f-\nabla p-\left(\left.a_{\varepsilon}\right|_{t=0}\right) P_{k}+\left(1-\varepsilon^{2} / \nu\right)\left(\left.a_{\varepsilon}\right|_{t=0}\right) P_{k} \\
& +\left(\Delta_{y} a-\nabla_{y} b-a_{t}^{\prime}\right)_{\varepsilon} * P_{k}+\left(1-\varepsilon^{2} / \nu\right)\left(a_{t}^{\prime}\right)_{\varepsilon} * P_{k} \\
& -\left(\nabla_{y} B\right)_{\varepsilon} P_{k}+2 \varepsilon\left(\nabla_{y} a\right)_{\varepsilon} * \nabla P_{k}+\varepsilon^{2} a_{\varepsilon} * \Delta P_{k} \\
& -\varepsilon b_{\varepsilon} * \nabla P_{k}-\varepsilon B_{\varepsilon} \nabla P_{k}+\varepsilon\left(\Delta_{y} w^{s}\right)_{\varepsilon} * \nabla P_{k} \\
& +2 \varepsilon^{2}\left(\nabla y w^{s}\right)_{\varepsilon} * \nabla \nabla P_{k}+\varepsilon^{3} w_{\varepsilon}^{s} * \Delta \nabla P_{k} \\
& -\varepsilon\left(\varepsilon^{2} / \nu\right) w_{\varepsilon}^{s} * \nabla\left(P_{k}\right)_{t}^{\prime} .
\end{aligned}
$$

Пусть $\varepsilon A_{\varepsilon}^{s k}$ обозначает последние восемь слагаемых справа в равенстве $(2.45)$ и $D_{k}=f-\nabla p-P_{k}, B_{\varepsilon}^{s k}=\left(\left.a_{\varepsilon}\right|_{t=0}\right) P_{k}+\left(a_{t}^{\prime}\right)_{\varepsilon} * P_{k}$. Тогда, используя $(0.2),(0.8)$ и неравенства (2.24), заключаем, что вьполнены равенство (2.11) (с $\varepsilon^{2} / \nu$, замененным на $\left.1-\varepsilon^{2} / \nu\right)$ и неравенства (2.12). Следовательно, учитывая $(2.25),(2.42),(2.43)$ и повторяя окончание доказательства теоремы 1 , получаем при $\varepsilon \rightarrow 0$ соотношение $(0.7)$ и слабую сходимость последовательности $\left\{\left(\nu / \varepsilon^{2}\right) u_{\varepsilon}\right\}$ к $A_{2} *(f-\nabla p)$ в $L^{2}\left(0, T ; L^{2}(\Omega)^{m}\right)$. В силу условия $(0.2)$ последовательности $\left\{u_{\varepsilon}\right\}$ и $\left\{\left(\nu / \varepsilon^{2}\right) u_{\varepsilon}\right\}$ имеют совпадающие слабые пределы.

Таким образом, для завершения доказательства теоремы 2 остается доказать, что $u_{\varepsilon} \rightarrow u$ слабо-* в $L^{\infty}\left(0, T ; L^{2}(\Omega)^{m}\right)$, если $f \in L^{1}\left(0, T ; L^{2}(\Omega)^{m}\right)$. Из леммы 4 имеем $\left\|u_{\varepsilon}\right\|_{L^{\infty}\left(0, T ; L^{2}(\Omega)^{m}\right)} \leqslant C$, и поэтому [3] некоторая подпоследовательность последовательности $\left\{u_{\varepsilon}\right\}$ сходится слабо- ${ }^{*} L^{\infty}\left(0, T ; L^{2}(\Omega)^{m}\right)$ к некоторому пределу $v$. Следовательно, повторяя доказательство (2.18), заключаем, что $v=A_{2} *(f-\nabla p)$, и теорема 2 доказана. 
ДОКАЗАТЕЛЬСТВО ТЕОРЕМЫ 3. В соответствии с результатами [1], [2] решение $B(y)$ задачи $(0.10)$ с компонентами из $H_{P}^{1}(F) \cap L^{2}(F) / \mathbb{R}$ существует и единственно. Следовательно, $a(y)=E-\nabla B(y) \in L^{2}(F)^{m \times m}$. Определим $a_{\varepsilon}=a(x / \varepsilon)$, $B_{\varepsilon}=B(x / \varepsilon),\left(\nabla_{y} B\right)_{\varepsilon}=\left(\nabla_{y} B\right)(x / \varepsilon)$ и продолжим эти функции по $\varepsilon$-периодичности на $\Omega_{\varepsilon}$. Тогда

$$
\left\|a_{\varepsilon}\right\|_{L^{2}(\Omega)^{m \times m}} \leqslant C, \quad\left\|B_{\varepsilon}\right\|_{L^{2}(\Omega)^{m}} \leqslant C .
$$

Известно [12], что $A_{3}=\langle a\rangle$ является симметричной, положительно определенной матрицей с постоянными компонентами. Кроме того, найдется такое $W_{\varepsilon} \in$ $H^{1}(\Omega)^{m \times m \times m}$, что $\left\|W_{\varepsilon}\right\|_{L^{2}(\Omega)^{m \times m \times m}} \leqslant C$ и вьполнено соотношение (2.2). Здесь и далее при ссылках на формулы из доказательства теоремы 1 предполагается, что $A_{1}$ в этих формулах заменено на $A_{3}$. Аналогично, найдется такое $w \in$ $H_{P}^{1}(F, S)^{m \times m \times m}$, что $\operatorname{div}_{y} w=A_{3} /|F|-a$. Далее, сушествует последовательность $\left\{w^{s}\right\} \subset C_{P}^{\infty}(F, S)^{m \times m \times m}$, сходящаяся к $w$ в норме $H_{P}^{1}(F, S)^{m \times m \times m}$. Определим $w_{\varepsilon}^{s}=w^{s}(x / \varepsilon),\left(\operatorname{div}_{y} w^{s}\right)_{\varepsilon}=\left(\operatorname{div}_{y} w^{s}\right)(x / \varepsilon)$, продолжим эти функции по $\varepsilon$-периодичности на $\Omega_{\varepsilon}$ и $w_{\varepsilon}^{s}$ нулем на $\Omega \backslash \Omega_{\varepsilon}$. Тогда равномерно по $\varepsilon$ выполнено соотношение (2.3).

В соответствии с $(0.10)$ имеем $\operatorname{div}_{y} a=0$ в $F$ и $(a, N)=0$ на $S$. Из леммы 6 следует, что найдется последовательность $\left\{a_{l}\right\} \subset C_{P}^{\infty}(F, S)^{m \times m}$ соленоидальных вектор-функций, сходящаяся к $a$ в норме $L^{2}(F)^{m \times m}$. Определим $a_{\varepsilon}^{l}=$ $a_{l}(x / \varepsilon)$, продолжим эти функции по $\varepsilon$-периодичности на $\Omega_{\varepsilon}$ и нулем на $\Omega \backslash \Omega_{\varepsilon}$. Тогда $\operatorname{div} a_{\varepsilon}^{l}=0$ для каждого $l=1,2, \ldots$ и равномерно по $\varepsilon$ выполнено соотношение

$$
\left\|a_{\varepsilon}^{l}-a_{\varepsilon}\right\|_{L^{2}\left(\Omega_{\varepsilon}\right)^{m \times m}} \leqslant C\left\|a_{l}-a\right\|_{L^{2}(F)^{m \times m}} \rightarrow 0 \text { при } l \rightarrow \infty .
$$

Осредненная задача для "предельного" давления $p$ в рассматриваем случае имеет вид

$$
\operatorname{div} A_{3} \int_{0}^{t}(f-\nabla p) d t=0 \quad \text { в } \Omega, \quad\left(A_{3} \int_{0}^{t}(f-\nabla p) d t, n\right)=0 \text { на } \partial \Omega .
$$

Разумеется, эти соотношения можно продифференцировать по $t$ и предположить (например, по определению), что $p$ является решением задачи (0.11) с $A_{1}$, замененным на $A_{3}$. Тогда существование и единственность решения $p \in L^{2}\left(0, T ; H^{1}(\Omega) \cap\right.$ $\left.L^{2}(\Omega) / \mathbb{R}\right)$ этой задачи следует из положительной определенности матрицы $A_{3}$. Пусть $G=A_{3}(f-\nabla p)$. В силу леммы 7 найдется последовательность $\left\{G_{k}\right\} \subset$ $C_{0}^{\infty}((0, T) \times \Omega)^{m}$ соленоидальных вектор-функций, сходяшаяся к $G$ в норме $L^{2}\left(0, T ; L^{2}(\Omega)^{m}\right)$. Пусть $P_{k}=A_{3}^{-1} G_{k}$. Тогда выполнены равенство $(2.5)$ и соотношение (2.6).

Используя введенные выше функции, определим последовательности $v_{\varepsilon}^{s l k}$ и $q_{\varepsilon}^{k}$, “приближаюшие" решение задачи (0.4):

$$
v_{\varepsilon}^{s l k}=a_{\varepsilon}^{l} \int_{0}^{t} P_{k} d t+\varepsilon w_{\varepsilon}^{s} \nabla \int_{0}^{t} P_{k} d t, \quad q_{\varepsilon}^{k}=p+\varepsilon B_{\varepsilon} P_{k} .
$$

Учитывая (2.5) и соленоидальность $a_{\varepsilon}^{l}$, получаем равенство

$$
\begin{aligned}
\operatorname{div} v_{\varepsilon}^{s l k}= & \left(\left(\operatorname{div}_{y} w^{s}\right)_{\varepsilon}-A_{3} /|F|+a_{\varepsilon}\right) \nabla \int_{0}^{t} P_{k} d t \\
& +\left(a_{\varepsilon}^{l}-a_{\varepsilon}\right) \nabla \int_{0}^{t} P_{k} d t+\varepsilon w_{\varepsilon}^{s} \nabla \nabla \int_{0}^{t} P_{k} d t .
\end{aligned}
$$


Поскольку функции $w_{\varepsilon}^{s}$ и $P_{k}$ являются гладкими и конечное $T$ фиксировано, из $(0.4)$ и $(2.50)$ получаем неравенство

$$
\left\|\operatorname{div}\left(u_{\varepsilon}-v_{\varepsilon}^{s l k}\right)\right\|_{L^{2}\left(0, T ; L^{2}\left(\Omega_{\varepsilon}\right)\right)} \leqslant \delta_{s l} C_{k}+\varepsilon C_{s k},
$$

где $C_{s k}, \delta_{s l}$ не зависят от $\varepsilon$ и, кроме того, $\delta_{s l}$ достаточно мало при больших $s$ и $l$ в соответствии с (2.3) и (2.47). Кроме того, из условия (0.3) и (2.51) следует, что выполнено неравенство

$$
\left\|\operatorname{div}\left(u_{\varepsilon}-v_{\varepsilon}^{s l k}\right)\right\|_{L^{2}\left(0, T ; L^{2}\left(\Omega_{\varepsilon}\right)\right)} \leqslant \frac{\varepsilon^{2}}{\nu} \delta_{s l} C_{k}+\frac{\varepsilon^{3}}{\nu} C_{s k}
$$

с постоянными $C_{s k}, \delta_{s l}$ из неравенства (2.51). Аналогично, из (0.4) и (2.49) имеем равенство

$$
\begin{aligned}
\left(u_{\varepsilon}\right. & \left.-v_{\varepsilon}^{s l k}\right)_{t}^{\prime}-\nu \Delta\left(u_{\varepsilon}-v_{\varepsilon}^{s l k}\right)+\nabla\left(p_{\varepsilon}-q_{\varepsilon}^{k}\right)=f-\nabla p-\left(\nabla_{y} B\right)_{\varepsilon} P_{k} \\
& -a_{\varepsilon} P_{k}-\left(a_{\varepsilon}^{l}-a_{\varepsilon}\right) P_{k}+\left(\nu / \varepsilon^{2}\right) \Delta\left(\varepsilon^{2} v_{\varepsilon}^{s l k}\right)-\varepsilon B_{\varepsilon} \nabla P_{k}-\varepsilon w_{\varepsilon}^{s} \nabla P_{k} .
\end{aligned}
$$

Пусть $\varepsilon A_{\varepsilon}^{s k}$ обозначает последние два слагаемых в правой части равенства (2.53) и $D_{k}=f-\nabla p-P_{k}, D_{\varepsilon}^{l k}=\left(a_{\varepsilon}-a_{\varepsilon}^{l}\right) P_{k}, B_{\varepsilon}^{s l k}=\Delta\left(\varepsilon^{2} v_{\varepsilon}^{s l k}\right)$. Тогда в силу определения $a_{\varepsilon}=E-\left(\nabla_{y} B\right)_{\varepsilon}$ выполнено равенство

$$
\left(u_{\varepsilon}-v_{\varepsilon}^{s l k}\right)_{t}^{\prime}-\nu \Delta\left(u_{\varepsilon}-v_{\varepsilon}^{s l k}\right)+\nabla\left(p_{\varepsilon}-q_{\varepsilon}^{k}\right)=D_{k}+D_{\varepsilon}^{l k}+\varepsilon A_{\varepsilon}^{s k}+\left(\nu / \varepsilon^{2}\right) B_{\varepsilon}^{s l k} .
$$

Используя (2.46) и гладкость $a_{\varepsilon}^{l}, w_{\varepsilon}^{s}, P_{k}$, имеем неравенства

$$
\begin{aligned}
\left\|A_{\varepsilon}^{s k}\right\|_{L^{2}\left(0, T ; L^{2}\left(\Omega_{\varepsilon}\right)^{m}\right)} & \leqslant C_{s k}, \quad\left\|B_{\varepsilon}^{s l k}\right\|_{L^{2}\left(0, T ; L^{2}\left(\Omega_{\varepsilon}\right)^{m}\right)} \leqslant C_{s l k}, \\
\left\|D_{k}\right\|_{L^{2}\left(0, T ; L^{2}\left(\Omega_{\varepsilon}\right)^{m}\right)} & \leqslant \delta_{k}, \quad\left\|D_{\varepsilon}^{l k}\right\|_{L^{2}\left(0, T ; L^{2}\left(\Omega_{\varepsilon}\right)^{m}\right)} \leqslant \delta_{l} C_{k},
\end{aligned}
$$

где $C_{s l k}, C_{s k}, C_{k}, \delta_{k}, \delta_{l}$ не зависят от $\varepsilon$ и, кроме того, $\delta_{k}$ и $\delta_{l}$ достаточно малы при больших $k$ и $l$ в соответствии с (2.6) и (2.47).

Учитывая $(2.52),(2.55)$ и повторяя доказательство неравенств $(2.15),(2.16)$, получаем оценку

$$
\begin{aligned}
& \left\|\left(u_{\varepsilon}-v_{\varepsilon}^{s l k}\right)_{t}^{\prime}\right\|_{L^{2}\left(0, T ; L^{2}(\Omega)^{m}\right)}+(\nu / \varepsilon)\left\|\nabla\left(u_{\varepsilon}-v_{\varepsilon}^{s k}\right)\right\|_{L^{2}\left(0, T ; L^{2}\left(\Omega_{\varepsilon}\right)^{m \times m}\right)}^{2} \\
& \quad \leqslant \sqrt{\varepsilon} C_{s k}+\delta_{s l} C_{k}+\delta_{s} C_{k}+\delta_{k} C+\left(\nu / \varepsilon^{2}\right) C_{s l k}
\end{aligned}
$$

Здесь и далее $C_{s l k}, C_{s k}, C_{k}, \delta_{s l}, \delta_{k}, \delta_{l}$ не зависят от $\varepsilon$ и, кроме того, $\delta_{s l}, \delta_{k}, \delta_{l}$ достаточно малы при больших $s, k$ и $l$. Таким образом, выполнен аналог неравенства (2.17), из которого (при достаточно больших $k$, затем при достаточно больших $s, l$ и, наконец, при достаточно малых $\left.\varepsilon, \nu / \varepsilon^{2}\right)$ и следует соотношение (0.7) теоремы 3.

Умножая скалярно в $L^{2}\left(\Omega_{\varepsilon}\right)^{m}$ равенство $(2.54)$ на $u_{\varepsilon}-v_{\varepsilon}^{s l k}$ и интегрируя полученное соотношение по $(0, t)$, имеем (см. (1.2)) неравенства

$$
\begin{aligned}
\| u_{\varepsilon}- & v_{\varepsilon}^{s l k} \|_{L^{\infty}\left(0, T ; L^{2}(\Omega)^{m}\right)}^{2} \leqslant 2 \int_{0}^{T}\left(p_{\varepsilon}-q_{\varepsilon}^{k}, \operatorname{div}\left(u_{\varepsilon}-v_{\varepsilon}^{s l k}\right)\right)_{L^{2}\left(\omega_{k} \cap \Omega_{\varepsilon}\right)} d t \\
& +2 \int_{0}^{T}\left(D_{k}+D_{\varepsilon}^{l k}+\varepsilon A_{\varepsilon}^{s k}+\left(\nu / \varepsilon^{2}\right) B_{\varepsilon}^{s l k},\left(u_{\varepsilon}-v_{\varepsilon}^{s l k}\right)\right)_{L^{2}\left(\Omega_{\varepsilon}\right)^{m}} d t \\
& \leqslant \varepsilon C_{s k}+\delta_{s l} C_{k}+\left(\delta_{k}+\delta_{l} C_{k}+\varepsilon C_{s k}+\left(\nu / \varepsilon^{2}\right) C_{s l k}\right)\left\|u_{\varepsilon}-v_{\varepsilon}^{s k}\right\|_{L^{\infty}\left(0, T ; L^{2}(\Omega)^{m}\right)},
\end{aligned}
$$


где учтены неравенства $(2.13),(2.51),(2.55)$ и конечность фиксированного T. Учитывая $\varepsilon$-неравенство, из (2.57) получаем оценку

$$
\left\|u_{\varepsilon}-v_{\varepsilon}^{s k}\right\|_{L^{\infty}\left(0, T ; L^{2}(\Omega)^{m}\right)} \leqslant \sqrt{\varepsilon} C_{s k}+\delta_{s l} C_{k}+\delta_{s} C_{k}+\delta_{k} C+\left(\nu / \varepsilon^{2}\right) C_{s l k} .
$$

Повторяя доказательство соотношения (2.18), из неравенств (2.56), (2.58) (при достаточно больших $k, s, l$ и при достаточно малых $\left.\varepsilon, \nu / \varepsilon^{2}\right)$ заключаем, что

$$
u_{\varepsilon} \rightarrow A_{3} \int_{0}^{t}(f-\nabla p) d t \quad \text { слабо- }{ }^{*} \text { в } L^{\infty}\left(0, T ; L^{2}(\Omega)^{m}\right)
$$

и $\left(u_{\varepsilon}\right)_{t}^{\prime} \rightarrow A_{3}(f-\nabla p)$ слабо в $L^{2}\left(0, T ; L^{2}(\Omega)^{m}\right)$. Теорема 3 доказана.

ДокАЗАТЕЛЬСТво теОремы 4. Повторяем доказательство теоремы 3 до равенства (2.50), заменяя $p$ на $q$ в задаче (2.48). Учитьвая, что $f \in L^{1}\left(0, T ; L^{2}(\Omega)^{m}\right)$, для решения этой задачи имеем $q \in L^{1}\left(0, T ; H^{1}(\Omega) \cap L^{2}(\Omega) / \mathbb{R}\right) \cap L^{2}\left(0, T ; H^{1}(\Omega) \cap\right.$ $\left.L^{2}(\Omega) / \mathbb{R}\right)$. Поскольку функции $w_{\varepsilon}^{s}$ и $P_{k}$ являются гладкими и финитными, из $(0.4)$ и (2.50) получаем неравенство

$$
\left\|\operatorname{div}\left(v_{\varepsilon}-v_{\varepsilon}^{s l k}\right)_{t}^{\prime}\right\|_{L^{2}\left(0, T ; L^{2}\left(\Omega_{\varepsilon}\right)\right)} \leqslant \delta_{s l} C_{k}+\varepsilon C_{s k}
$$

Здесь и далее $C_{s k}, C_{k}, \delta_{s l}$ не зависят от $\varepsilon$ и, кроме того, $\delta_{s l}$ достаточно мало при больших $s$ и $l$ в соответствии с (2.3) и (2.47). Аналогично, из (0.13) и (2.49) следует равенство

$$
\left(v_{\varepsilon}-v_{\varepsilon}^{s l k}\right)_{t}^{\prime}+\nabla\left(q_{\varepsilon}-q_{\varepsilon}^{k}\right)=D_{k}+D_{\varepsilon}^{l k}+\varepsilon A_{\varepsilon}^{s k}
$$

где $A_{\varepsilon}^{s k}=-B_{\varepsilon} \nabla P_{k}-w_{\varepsilon}^{s} \nabla P_{k}, D_{k}=f-\nabla p-P_{k}, D_{\varepsilon}^{l k}=\left(a_{\varepsilon}-a_{\varepsilon}^{l}\right) P_{k}$ удовлетворяют неравенствам (2.55). В этих неравенствах и далее $C_{s l k}, \delta_{k}, \delta_{l}$ не зависят от $\varepsilon$ и, кроме того, $\delta_{k}$ и $\delta_{l}$ достаточно малы при больших $k$ и $l$ в соответствии с (2.6) и $(2.47)$.

Умножим скалярно в $L^{2}\left(\Omega_{\varepsilon}\right)^{m}$ первое уравнение $(0.13)$ на $\nabla q_{\varepsilon}$. Тогда для п.в. $t$ имеем неравенство

$$
\left\|\nabla q_{\varepsilon}\right\|_{L^{2}\left(\Omega_{\varepsilon}\right)^{m}} \leqslant\|f\|_{L^{2}\left(\Omega_{\varepsilon}\right)^{m}}
$$

Следовательно, $\nabla q_{\varepsilon} \in L^{1}\left(0, T ; L^{2}\left(\Omega_{\varepsilon}\right)\right) \cap L^{2}\left(0, T ; L^{2}\left(\Omega_{\varepsilon}\right)\right)$ и выполнено неравенство $(2.13)$ с $p_{\varepsilon}$, замененным на $q_{\varepsilon}$. Умножая скалярно в $L^{2}\left(0, T ; L^{2}\left(\Omega_{\varepsilon}\right)^{m}\right)$ равенство $(2.60)$ на $\left(v_{\varepsilon}-v_{\varepsilon}^{s l k}\right)_{t}^{\prime}$, получаем оценку

$$
\begin{gathered}
\left\|\left(v_{\varepsilon}-v_{\varepsilon}^{s l k}\right)_{t}^{\prime}\right\|_{L^{2}\left(0, T ; L^{2}\left(\Omega_{\varepsilon}\right)^{m}\right)}^{2} \leqslant \int_{0}^{T}\left(q_{\varepsilon}-q_{\varepsilon}^{k}, \operatorname{div}\left(v_{\varepsilon}-v_{\varepsilon}^{s l k}\right)_{t}^{\prime}\right)_{L^{2}\left(\omega_{k} \cap \Omega_{\varepsilon}\right)} d t \\
+\int_{0}^{T}\left(D_{k}+D_{\varepsilon}^{l k}+\varepsilon A_{\varepsilon}^{s k},\left(v_{\varepsilon}-v_{\varepsilon}^{s l k}\right)_{t}^{\prime}\right)_{L^{2}\left(\Omega_{\varepsilon}\right)^{m}} d t
\end{gathered}
$$

из которой и из $(2.13),(2.55),(2.59)$ следует неравенство

$$
\left\|\left(v_{\varepsilon}-v_{\varepsilon}^{s l k}\right)_{t}^{\prime}\right\|_{L^{2}\left(0, T ; L^{2}\left(\Omega_{\varepsilon}\right)^{m}\right)} \leqslant \sqrt{\varepsilon} C_{s k}+\delta_{s l} C_{k}+\delta_{l} C_{k}+\delta_{k} C .
$$


Следовательно, используя леммы 2,3 и равенство $(2.60)$, для $\omega \subset \subset \Omega$ имеем неравенства

$$
\begin{aligned}
\| q_{\varepsilon} & -q_{\varepsilon}^{k}\left\|_{L^{2}\left(0, T ; L^{2}\left(\omega \cap \Omega_{\varepsilon}\right)\right)} \leqslant(C / \varepsilon)\right\| \nabla\left(q_{\varepsilon}-q_{\varepsilon}^{k}\right) \|_{L^{2}\left(0, T ; H^{-1}\left(\Omega_{\varepsilon}\right)^{m}\right)} \\
& \leqslant C\left\|\nabla\left(q_{\varepsilon}-q_{\varepsilon}^{k}\right)\right\|_{L^{2}\left(0, T ; L^{2}\left(\left(\Omega_{\varepsilon}\right)^{m}\right)\right.} \\
& =C\left\|D_{k}+D_{\varepsilon}^{l k}+\varepsilon A_{\varepsilon}^{s k}-\left(v_{\varepsilon}-v_{\varepsilon}^{s l k}\right)_{t}^{\prime}\right\|_{L^{2}\left(0, T ; L^{2}\left(\Omega_{\varepsilon}\right)^{m}\right)} \\
& \leqslant \sqrt{\varepsilon} C_{s k}+\delta_{s l} C_{k}+\delta_{l} C_{k}+\delta_{k} C
\end{aligned}
$$

где учтены неравенства (2.55) и (2.62). Из неравенств (2.62) и (2.63), как и в доказательстве теоремы 1 , выводятся утверждения теоремы 4 о сходимости $q_{\varepsilon}$ и $\left(v_{\varepsilon}\right)_{t}^{\prime}$ к соответствуюшим пределам. Однако для доказательства утверждения о сходимости $v_{\varepsilon}$ потребуются дополнительные рассуждения.

Предположим в условиях леммы 7 , что $u \in L^{1}(0, T ; H(\Omega))$. Тогда найдется последовательность $\left\{u_{k}\right\} \subset C_{0}^{\infty}((0, \infty) \times \Omega)^{m}$ соленоидальных вектор-функций, сходящаяся к $u$ в норме $L^{1}(0, T ; H(\Omega))$. Действительно, в соответствии с [21, гл. $6, \S 7]$ в $L^{1}(0, T ; H(\Omega))$ плотно множество ступенчатых функций. Каждая ступенчатая функция приближается конечной линейной комбинацией векторов вида $a_{s} \varphi_{l}$ с $\left\{a_{s}\right\} \in C_{0}^{\infty}(0, \infty), \varphi_{l} \in V(\Omega)$, что и требуется доказать. Таким образом, заменяя при необходимости $P_{k}$ в $(2.49)$, получаем неравенства

$$
\begin{gathered}
\left\|A_{\varepsilon}^{s k}\right\|_{L^{1}\left(0, T ; L^{2}\left(\Omega_{\varepsilon}\right)^{m}\right)} \leqslant C_{s k}, \quad\left\|D_{k}\right\|_{L^{1}\left(0, T ; L^{2}\left(\Omega_{\varepsilon}\right)^{m}\right)} \leqslant \delta_{k}, \\
\left\|D_{\varepsilon}^{l k}\right\|_{L^{1}\left(0, T ; L^{2}\left(\Omega_{\varepsilon}\right)^{m}\right)} \leqslant \delta_{l} C_{k} .
\end{gathered}
$$

Повторяя доказательства лемм 2 и 3 , заключаем, что выполнено неравенство

$$
\left\|q_{\varepsilon}-q_{\varepsilon}^{k}\right\|_{L^{1}\left(0, T ; L^{2}\left(\omega_{k} \cap \Omega_{\varepsilon}\right)\right)} \leqslant C_{k}
$$

с соответствуюшим $\omega_{k} \subset \subset \Omega$ (см. доказательство (2.13)). Кроме того, из (0.13) и $(2.50)$ следует неравенство

$$
\left\|\operatorname{div}\left(v_{\varepsilon}-v_{\varepsilon}^{s l k}\right)\right\|_{L^{\infty}\left(0, T ; L^{2}\left(\Omega_{\varepsilon}\right)\right)} \leqslant \delta_{s l} C_{k}+\varepsilon C_{s k} .
$$

Отметим, что в силу определений $w_{\varepsilon}^{s}, P_{k}$ и $(2.50)$ неравенство (2.66) выполнено для произвольных $T$, в отличие от неравенства (2.51). Используя (2.64)-(2.66) при оценке соответствуюших интегралов в аналоге соотношения $(2.57)$, имеем неравенство

$$
\left\|v_{\varepsilon}-v_{\varepsilon}^{s l k}\right\|_{L^{\infty}\left(0, T ; L^{2}(\Omega)^{m}\right)} \leqslant \sqrt{\varepsilon} C_{s k}+\delta_{s l} C_{k}+\delta_{l} C_{k}+\delta_{k} C .
$$

Из этого неравенства, как и в доказательстве (2.18), выводим, что

$$
v_{\varepsilon} \rightarrow A_{3} \int_{0}^{t}(f-\nabla p) d t \quad \text { слабо- }^{*}{ }^{\text {в }} \quad L^{\infty}\left(0, T ; L^{2}(\Omega)^{m}\right),
$$

и теорема 4 доказана. 
ДОКАЗАТЕЛЬСТВО ТЕОРЕМЫ 5. Соотношения (0.14), (0.15) можно записать в виде краевой задачи

$$
v_{\varepsilon}+\nabla q_{\varepsilon}=w, \quad \operatorname{div} v_{\varepsilon}=0 \quad \text { в } \Omega_{\varepsilon}, \quad\left(v_{\varepsilon}, n_{\varepsilon}\right)=0 \text { на } \partial \Omega_{\varepsilon},
$$

где $w \in L^{2}(\Omega)^{m}$. Таким образом, имеется "стационарная" аналогия с задачей (0.13). Следовательно, повторяя начало доказательства теоремы 4 , получаем соотношения $(2.2),(2.3),(2.46)$ и (2.47). Осредненная задача для "предельного" давления $q$ в рассматриваем случае имеет вид

$$
\operatorname{div} A_{3}(w-\nabla q)=0 \quad \text { в } \Omega, \quad\left(A_{3}(w-\nabla q), n\right)=0 \quad \text { на } \partial \Omega .
$$

Сушествование и единственность решения $q \in H^{1}(\Omega) \cap L^{2}(\Omega) / \mathbb{R}$ этой задачи следует из положительной определенности матрицы $A_{3}$. Пусть $G=A_{3}(w-\nabla q)$. В силу плотности множества $V(\Omega)$ в $H(\Omega)$ (см. доказательство леммы 7 ) найдется последовательность $\left\{G_{k}\right\} \subset C_{0}^{\infty}(\Omega)^{m}$ соленоидальных вектор-функций, сходящаяся к $G$ в норме $L^{2}(\Omega)^{m}$. Пусть $P_{k}=A_{3}^{-1} G_{k}$. Тогда вьполнены равенство $(2.5)$ с $A_{1}$, замененным на $A_{3}$, и соотношение

$$
\left\|w-\nabla q-P_{k}\right\|_{L^{2}(\Omega)^{m}} \rightarrow 0 \text { при } k \rightarrow \infty .
$$

Используя введенные выше функции, определим последовательности $v_{\varepsilon}^{s l k}$ и $q_{\varepsilon}^{k}$, "приближающие" решение задачи (2.67):

$$
v_{\varepsilon}^{s l k}=a_{\varepsilon}^{l} P_{k}+\varepsilon w_{\varepsilon}^{s} \nabla P_{k}, \quad q_{\varepsilon}^{k}=p+\varepsilon B_{\varepsilon} P_{k} .
$$

Учитывая $(2.3),(2.5)$ и $(2.47)$, из (2.67) и (2.70) получаем неравенство

$$
\left\|\operatorname{div}\left(v_{\varepsilon}-v_{\varepsilon}^{s l k}\right)\right\|_{L^{2}\left(\Omega_{\varepsilon}\right)} \leqslant \delta_{s l} C_{k}+\varepsilon C_{s k} .
$$

Аналогично, из (2.67) и (2.70) имеем равенство

$$
v_{\varepsilon}-v_{\varepsilon}^{s l k}+\nabla\left(q_{\varepsilon}-q_{\varepsilon}^{k}\right)=D_{k}+D_{\varepsilon}^{l k}+\varepsilon A_{\varepsilon}^{s k}
$$

где $A_{\varepsilon}^{s k}=-B_{\varepsilon} \nabla P_{k}-w_{\varepsilon}^{s} \nabla P_{k}, D_{k}=f-\nabla p-P_{k}, D_{\varepsilon}^{l k}=\left(a_{\varepsilon}-a_{\varepsilon}^{l}\right) P_{k}$ удовлетворяют "стационарному варианту" неравенств (2.55). Кроме того, выполнено неравенство (2.61). Следовательно, повторяя доказательство неравенств (2.62) и (2.63), для $\omega \subset \subset \Omega$ имеем неравенства

$$
\begin{aligned}
\left\|v_{\varepsilon}-v_{\varepsilon}^{s l k}\right\|_{L^{2}(\Omega)^{m}} & \leqslant \sqrt{\varepsilon} C_{s k}+\delta_{s l} C_{k}+\delta_{l} C_{k}+\delta_{k} C, \\
\left\|q_{\varepsilon}-q_{\varepsilon}^{k}\right\|_{L^{2}\left(\omega \cap \Omega_{\varepsilon}\right)} & \leqslant C\left\|\nabla\left(q_{\varepsilon}-q_{\varepsilon}^{k}\right)\right\|_{L^{2}\left(\Omega_{\varepsilon}\right)^{m}} \\
& \leqslant \sqrt{\varepsilon} C_{s k}+\delta_{s l} C_{k}+\delta_{l} C_{k}+\delta_{k} C,
\end{aligned}
$$

где использованы соотношения $(2.55),(2.68),(2.71),(2.72)$ и неравенства из доказательства лемм 2 и 3 . Из неравенств (2.73), как и в доказательстве теоремы 1 , выводятся утверждения теоремы 5 о сходимости $q_{\varepsilon}$ и $v_{\varepsilon}$ к соответствующим пределам.

Пусть $u_{\varepsilon}$ и $p_{\varepsilon}$ являются решениями начально-краевой задачи $(0.4),(0.16)$. Определим

$$
\begin{gathered}
U_{\varepsilon}=\int_{0}^{t} u_{\varepsilon}(\tau) d \tau, \quad P_{\varepsilon}=\int_{0}^{t} p_{\varepsilon}(\tau) d \tau, \quad F=\int_{0}^{t} f(\tau) d \tau \\
\widetilde{P}_{\varepsilon}=P_{\varepsilon}+q_{\varepsilon}, \quad \widetilde{F}=F+\omega .
\end{gathered}
$$


Тогда в соответствии с [1] (см. также [7]) имеем для $U_{\varepsilon}$ и $\widetilde{P}_{\varepsilon}$ начально-краевую задачу

$$
\begin{gathered}
\left(U_{\varepsilon}\right)_{t}^{\prime}-\nu \Delta U_{\varepsilon}+\nabla \widetilde{P}_{\varepsilon}=\widetilde{F}, \quad \operatorname{div} U_{\varepsilon}=0 \text { в } \Omega_{\varepsilon} \times(0, T), \\
U_{\varepsilon}=0 \text { на } \partial \Omega_{\varepsilon} \times(0, T),\left.\quad U_{\varepsilon}\right|_{t=0}=0 \text { в } \Omega_{\varepsilon},
\end{gathered}
$$

где $\widetilde{F} \in L^{2}\left(0, T ; L^{2}(\Omega)^{m}\right)$, поскольку конечное $T$ фиксировано. Таким образом, для вычисления пределов последовательностей $\left\{U_{\varepsilon}\right\}$ и $\left\{\widetilde{P}_{\varepsilon}\right\}$ можно использовать теоремы 1,2 или 3.

Пусть выполнено условие (0.1). Тогда в силу теоремы 1 для $\omega \subset \subset \Omega$ при $\varepsilon \rightarrow 0$ имеем соотношения

$$
\begin{aligned}
\widetilde{P}_{\varepsilon} & \rightarrow \widetilde{P} \quad \text { в норме } \\
\left(\nu / \varepsilon^{2}\right) U_{\varepsilon} & \rightarrow A_{1}(\widetilde{F}-\nabla \widetilde{P}) \quad \text { слабо в } \quad L^{2}\left(0, T ; L^{2}(\Omega)^{m}\right),
\end{aligned}
$$

где $\widetilde{P}$ является решением краевой задачи

$$
\operatorname{div} A_{1}(\widetilde{F}-\nabla \widetilde{P})=0 \quad \text { в } \Omega, \quad\left(A_{1}(\widetilde{F}-\nabla \widetilde{P}), n\right)=0 \quad \text { на } \partial \Omega .
$$

Учитывая единственность решения и линейность задачи (2.77), получаем представление

$$
\widetilde{P}=s+\int_{0}^{t} p(\tau) d \tau
$$

где $p$ является решением краевой задачи (0.11) и $s$ удовлетворяет (2.68) с $A_{3}$, замененным на $A_{1}$. Непосредственно из определений и (2.76) получаем соотношение

$$
P_{\varepsilon}=\widetilde{P}_{\varepsilon}-q_{\varepsilon} \rightarrow s-q+\int_{0}^{t} p(\tau) d \tau \quad \text { в норме } \quad L^{2}\left(0, T ; L^{2}\left(\omega \cap \Omega_{\varepsilon}\right) / \mathbb{R}\right) .
$$

Но тогда $p=\left(P_{\varepsilon}\right)_{t}^{\prime}$ сходится к $p$ в норме $H^{-1}\left(0, T ; L^{2}\left(\omega \cap \Omega_{\varepsilon}\right) / \mathbb{R}\right)$. Пусть $\varphi \in$ $C_{0}^{\infty}((0, T) \times \Omega)^{m}$. Тогда $(\varphi)_{t}^{\prime} \in C_{0}^{\infty}((0, T) \times \Omega)^{m}$ и из $(2.76)$ следует, что

$$
\begin{aligned}
\int_{0}^{T}\left(\frac{\nu}{\varepsilon^{2}} u_{\varepsilon}, \varphi\right)_{L^{2}(\Omega)^{m}} d t=\int_{0}^{T}\left(\frac{\nu}{\varepsilon^{2}} U_{\varepsilon}, \varphi_{t}^{\prime}\right)_{L^{2}(\Omega)^{m}} d t \\
\quad \rightarrow \int_{0}^{T}\left(U, \varphi_{t}^{\prime}\right)_{L^{2}(\Omega)^{m}} d t=\int_{0}^{T}\left(A_{1}(f-\nabla p), \varphi\right)_{L^{2}(\Omega)^{m}} d t
\end{aligned}
$$

при $\varepsilon \rightarrow 0$, что и доказывает теорему $5,($ a).

Пусть выполнено условие (0.2). Тогда в силу теоремы 2 для $\omega \subset \subset \Omega$ при $\varepsilon \rightarrow 0$ имеем соотношения

$$
\begin{gathered}
\widetilde{P}_{\varepsilon} \rightarrow \widetilde{P} \quad \text { в норме } \\
L^{2}\left(0, T ; L^{2}\left(\omega \cap \Omega_{\varepsilon}\right) / \mathbb{R}\right), \\
U_{\varepsilon} \rightarrow A_{2} *(\widetilde{F}-\nabla \widetilde{P}) \quad \text { слабо в } \quad L^{2}\left(0, T ; L^{2}(\Omega)^{m}\right),
\end{gathered}
$$

где $\widetilde{P}$ является решением краевой задачи

$$
\operatorname{div} A_{2} *(\widetilde{F}-\nabla \widetilde{P})=0 \quad \text { в } \Omega, \quad\left(A_{2} *(\widetilde{F}-\nabla \widetilde{P}), n\right)=0 \text { на } \partial \Omega .
$$

Учитывая первое равенство из (2.41), продифференцируем соотношения (2.80) по $t$ и положим $t=0$. Тогда для $\left.\widetilde{P}\right|_{t=0}$ получим краевую задачу (2.68). Напомним, 
что $A_{2}(0)=A_{3}$. Следовательно, выполнено равенство $\left.\widetilde{P}\right|_{t=0}=q$. Учитывая второе равенство из (2.41), получаем соотношение

$$
U_{t}^{\prime}=A_{2}(w-\nabla q)+A_{2} *\left(f-\nabla \widetilde{P}_{t}^{\prime}\right) .
$$

Поскольку $\operatorname{div} U_{t}^{\prime}=0$ в $\Omega$ и $\left(U_{t}^{\prime}, n\right)=0$ на $\partial \Omega$, для $p=\widetilde{P}_{t}^{\prime}$ имеем краевую задачу $(0.17)$. Используя $(2.22)$ и повторяя доказательство сушествования единственного решения задачи $(2.27)$, заключаем, что $p \in L^{2}\left(0, T ; H^{1}(\Omega) \cap L^{2}(\Omega) / \mathbb{R}\right)$, удовлетворяющее (0.17), существует и единственно. Таким образом,

$$
P_{\varepsilon}=\widetilde{P}_{\varepsilon}-q_{\varepsilon} \rightarrow \int_{0}^{t} p(\tau) d \tau \quad \text { в норме } \quad L^{2}\left(0, T ; L^{2}\left(\omega \cap \Omega_{\varepsilon}\right) / \mathbb{R}\right)
$$

и $p_{\varepsilon}=\left(P_{\varepsilon}\right)_{t}^{\prime}$ сходится к $p$ в норме $H^{-1}\left(0, T ; L^{2}\left(\omega \cap \Omega_{\varepsilon}\right) / \mathbb{R}\right)$. Для начально-краевой задачи (0.4), (0.16) выполнено тождество (1.2). Следовательно, выполнено неравенство $\left\|u_{\varepsilon}\right\|_{L^{\infty}\left(0, T ; L^{2}(\Omega)^{m}\right)} \leqslant C$, и поэтому [3] некоторая подпоследовательность последовательности $\left\{u_{\varepsilon}\right\}$ сходится слабо- ${ }^{*} L^{\infty}\left(0, T ; L^{2}(\Omega)^{m}\right)$ к некоторому пределу $v$. Таким образом, повторяя доказательство $(2.78)$, заключаем, что $v=U_{t}^{\prime}$ и теорема 5, (b) доказана.

Доказательство теоремы $5,(\mathrm{c})$ вполне аналогично доказательствам в рассмотренных случаях. Теорема 5 доказана.

Пусть $v_{\varepsilon}$ определено равенством (0.15) и выполнено условие (0.1). Рассмотрим задачу (0.4) с "мальм" неоднородным начальным условием

$$
\left.u_{\varepsilon}\right|_{t=0}=(\varepsilon / \sqrt{\nu}) v_{\varepsilon} \quad \text { в } \quad \Omega_{\varepsilon} .
$$

Из соотношения (1.2) для $u_{\varepsilon}$, удовлетворяюшего (0.4), (2.81), имеем неравенство

$$
2 \nu \int_{0}^{T}\left\|\nabla u_{\varepsilon}\right\|_{L^{2}\left(\Omega_{\varepsilon}\right)^{m \times m}}^{2} d t \leqslant 2 \int_{0}^{T}\left(f, u_{\varepsilon}\right)_{L^{2}\left(\Omega_{\varepsilon}\right)^{m}} d t+\left(\varepsilon^{2} / \nu\right)\left\|v_{\varepsilon}\right\|_{L^{2}\left(\Omega_{\varepsilon}\right)^{m}}^{2} .
$$

Следовательно, как и в лемме 4 , получаем оценки

$$
(\nu / \varepsilon)\left\|\nabla u_{\varepsilon}\right\|_{L^{2}\left(0, T ; L^{2}(\Omega)^{m \times m}\right)} \leqslant C, \quad\left(\nu / \varepsilon^{2}\right)\left\|u_{\varepsilon}\right\|_{L^{2}\left(0, T ; L^{2}(\Omega)^{m}\right)} \leqslant C .
$$

Тогда некоторая подпоследовательность последовательности $\left\{\left(\nu / \varepsilon^{2}\right) u_{\varepsilon}\right\}$ слабо сходится в $L^{2}\left(0, T ; L^{2}(\Omega)^{m}\right)$ к некоторому пределу. Поэтому, повторяя доказательство (2.78), выводим для решения $u_{\varepsilon}$ задачи $(0.4),(2.81)$ при $\varepsilon \rightarrow 0$ соотношение

$$
\left(\nu / \varepsilon^{2}\right) u_{\varepsilon} \rightarrow A_{1}(f-\nabla p) \quad \text { слабо в } \quad L^{2}\left(0, T ; L^{2}(\Omega)^{m}\right),
$$

где $p$ является решением краевой задачи (0.11).

ДоКАЗАТЕЛЬСТво ТЕОРЕМЫ 6 . Условие (0.19) подразумевает, что выполнено (0.1). Повторяем доказательство теоремы 1 до равенства (2.10). Пусть $U_{\varepsilon}, P_{\varepsilon}$ и $F$ определены равенствами (2.74). Тогда

$$
\begin{gathered}
-\nu \Delta U_{\varepsilon}+\nabla P_{\varepsilon}=F-u_{\varepsilon}+(\varepsilon / \sqrt{\nu}) v_{\varepsilon}-\int_{0}^{t} u_{\varepsilon} \nabla u_{\varepsilon} d t, \\
\operatorname{div} U_{\varepsilon}=0 \text { в } \Omega_{\varepsilon} \times(0, T), \\
U_{\varepsilon}=0 \quad \text { на } \partial \Omega_{\varepsilon} \times(0, T),\left.\quad U_{\varepsilon}\right|_{t=0}=0 \text { в } \Omega_{\varepsilon} .
\end{gathered}
$$


В соответствии с [1], [2] решение $u_{\varepsilon}$ задачи $(0.18)$ удовлетворяет неравенству $(2.82)$ и поэтому оценкам (2.83). Пусть $v \in H_{0}^{1}\left(\Omega_{\varepsilon}\right)$. Тогда

$$
\left|\int_{\Omega_{\varepsilon}} u_{\varepsilon} \nabla u_{\varepsilon} v d x\right|=\left|\int_{\Omega_{\varepsilon}} u_{\varepsilon} \nabla v u_{\varepsilon} d x\right| \leqslant C\left\|u_{\varepsilon}\right\|_{L^{4}\left(\Omega_{\varepsilon}\right)^{m}}^{2}\|\nabla v\|_{L^{2}\left(\Omega_{\varepsilon}\right)^{m \times m}}
$$

Следовательно, используя известную оценку $L^{4}$-нормы для трехмерных областей $[2$, гл. $1, \S 1]$, получаем неравенства

$$
\left\|u_{\varepsilon} \nabla u_{\varepsilon}\right\|_{H^{-1}\left(\Omega_{\varepsilon}\right)^{m}} \leqslant C\left\|u_{\varepsilon}\right\|_{L^{4}\left(\Omega_{\varepsilon}\right)^{m}}^{2} \leqslant C\left\|u_{\varepsilon}\right\|_{L^{2}\left(\Omega_{\varepsilon}\right)^{m}}^{1 / 2}\left\|\nabla u_{\varepsilon}\right\|_{L^{2}\left(\Omega_{\varepsilon}\right)^{m \times m}}^{3 / 2}
$$

(в двумерном случае $\left\|u_{\varepsilon} \nabla u_{\varepsilon}\right\|_{H^{-1}\left(\Omega_{\varepsilon}\right)^{m}} \leqslant C\left\|u_{\varepsilon}\right\|_{L^{2}\left(\Omega_{\varepsilon}\right)^{m}}\left\|\nabla u_{\varepsilon}\right\|_{L^{2}\left(\Omega_{\varepsilon}\right)^{m \times m}}$ ). Таким образом,

$$
\begin{aligned}
& \varepsilon^{-1}\left\|\int_{0}^{t} u_{\varepsilon} \nabla u_{\varepsilon} d t\right\|_{H^{-1}\left(\Omega_{\varepsilon}\right)^{m}} \leqslant C \varepsilon^{-1} \int_{0}^{T}\left\|u_{\varepsilon} \nabla u_{\varepsilon}\right\|_{H^{-1}\left(\Omega_{\varepsilon}\right)^{m}} d t \\
& \quad \leqslant C \varepsilon^{-1} \int_{0}^{T}\left\|u_{\varepsilon}\right\|_{L^{2}\left(\Omega_{\varepsilon}\right)^{m}}^{1 / 2}\left\|\nabla u_{\varepsilon}\right\|_{L^{2}\left(\Omega_{\varepsilon}\right)^{m \times m}}^{3 / 2} d t \\
& \quad \leqslant C \varepsilon^{-1}\left\|u_{\varepsilon}\right\|_{L^{2}\left(0, T ; L^{2}(\Omega)^{m}\right)}^{1 / 2}\left\|\nabla u_{\varepsilon}\right\|_{L^{2}\left(0, T ; L^{2}(\Omega)^{m \times m}\right)}^{3 / 2} \leqslant C \varepsilon^{3 / 2} / \nu^{2},
\end{aligned}
$$

где учтены неравенства (2.83) (в двумерном случае величина $\varepsilon^{3 / 2} / \nu^{2}$ заменяется на $\left.\varepsilon^{2} / \nu^{2}\right)$. Используя неравенства $(2.83),(2.85)$ и повторяя окончание доказательства теоремы 1 , устанавливаем для $U_{\varepsilon}, P_{\varepsilon}$ соотношения $(2.76)$, из которых и следуют утверждения теоремы 6.

ДОКАЗАТЕЛЬСТВо тЕОРЕМЫ 7. В соответствии с [1], [2] решение $u_{\varepsilon}$ задачи $(0.20)$ удовлетворяет неравенству $\nu\left\|\nabla u_{\varepsilon}\right\|_{L^{2}\left(\Omega_{\varepsilon}\right)^{m \times m}}^{2} \leqslant\left(f, u_{\varepsilon}\right)_{L^{2}\left(\Omega_{\varepsilon}\right)^{m}}$ и поэтому оценкам

$$
(\nu / \varepsilon)\left\|\nabla u_{\varepsilon}\right\|_{L^{2}(\Omega)^{m \times m}} \leqslant C, \quad\left(\nu / \varepsilon^{2}\right)\left\|u_{\varepsilon}\right\|_{L^{2}(\Omega)^{m}} \leqslant C .
$$

Следовательно, учитывая (2.84), получаем неравенство

$$
\varepsilon^{-1}\left\|u_{\varepsilon} \nabla u_{\varepsilon}\right\|_{H^{-1}\left(\Omega_{\varepsilon}\right)^{m}} \leqslant C \varepsilon^{3 / 2} / \nu^{2}
$$

и для доказательства теоремы 7 достаточно повторить “стационарный" вариант доказательства теоремы 6 .

Проверим единственность решения задачи $(0.20)$ в случае, когда выполнено условие (0.19). Пусть $u_{\varepsilon}^{1}$ и $u_{\varepsilon}^{2}$ - два решения этой задачи. Тогда для $u_{\varepsilon}=u_{\varepsilon}^{1}-u_{\varepsilon}^{2}$ имеем неравенства

$$
\begin{aligned}
& \nu\left\|\nabla u_{\varepsilon}\right\|_{L^{2}\left(\Omega_{\varepsilon}\right)^{m \times m}}^{2}=-\int_{\Omega_{\varepsilon}} u_{\varepsilon} \nabla u_{\varepsilon}^{1} u_{\varepsilon} d x \\
& \quad \leqslant C\left\|u_{\varepsilon}\right\|_{L^{4}\left(\Omega_{\varepsilon}\right)^{m}}^{2}\left\|\nabla u_{\varepsilon}^{1}\right\|_{L^{2}\left(\Omega_{\varepsilon}\right)^{m \times m}} \leqslant C\left(\varepsilon^{3 / 2} / \nu\right)\left\|\nabla u_{\varepsilon}\right\|_{L^{2}\left(\Omega_{\varepsilon}\right)^{m \times m}}^{2},
\end{aligned}
$$

где учтены оценки (2.86) для $u_{\varepsilon}^{1}$, неравенства из доказательства леммы 1 и (2.84). Следовательно,

$$
\left(1-\left(\varepsilon^{3 / 2} / \nu^{2}\right) C\right)\left\|\nabla u_{\varepsilon}\right\|_{L^{2}\left(\Omega_{\varepsilon}\right)^{m \times m}}^{2} \leqslant 0
$$

и $\left\|\nabla u_{\varepsilon}\right\|_{L^{2}\left(\Omega_{\varepsilon}\right)^{m \times m}}=0$ в силу (0.19). Таким образом, для достаточно малых $\varepsilon$ решение $u_{\varepsilon}$ задачи $(0.20)$ определено однозначно. Единственность решения $u_{\varepsilon}$ задачи (0.18) доказывается аналогично.

Отметим в заключение, что в случае, когда множество $\square \backslash F$ имеет положительную меру Лебега в $\mathbb{R}^{m}$, или в случае, когда множество $S$ имеет положительную меру Лебега на $\partial F$, неравенство (0.6) доказано в [22]. 


\section{Список литературы}

1. Темам Р. Уравнения Навье-Стокса. М.: Мир, 1981.

2. Ладыженская O.A. Математические вопросы динамики вязкой несжимаемой жидкости. М.: Наука, 1970.

3. Санчес-Паленсия Э. Неоднородные среды и теория колебаний. М.: Мир, 1984.

4. Allaire $G$. Homogenization of the Stokes flow in a connected porous medium // Asymptotic Anal. 1989. V. 2. P. 203-222.

5. Бахвалов Н.С., Панасенко Г.П. Осреднение процессов в периодических средах. М.: Наука, 1984.

6. Жиков В.В. Об усреднении системы уравнений Стокса в перфорированной области // ДАН СССР. 1994. Т. 334. № 2. С. 144-147.

7. Mikelić A. Homogenization of nonstationary Navier-Stokes equations in a domain with grained boundary // Annali di Matematica pure ed applicata. 1991. V. CLVIII. P. 167-179.

8. Mikelić A. Mathematical derivation of the Darcy-type law with memory effects, governing transient flow through porous media // Glasnik Mat. 1994. V. 29. P. 57-77.

9. Allaire G. Homogenization of the unsteady Stokes equations in porous medium // Progress in partial differential equations: calculus of variations, applications. London: Longman Scientific and Technical, 1992.

10. Сандраков Г.В. Осреднение линеаризованной системы гидродинамики с малой вязкостью и скорость звука в смесях. Препринт № 178. М.: ОВМ АН СССР, 1987.

11. Сандраков Г.В. Осреднение нестационарного потока Стокса в периодической перфорированной среде // ДАН. 1996. Т. 347. № 3. С. 312-315.

12. Олейник О.А., Иосифьян Г.А., Шамаев А.С. Математические задачи теории сильно неоднородных упругих сред. М.: Изд-во МГУ, 1990.

13. Валединский В.Д., Кобельков Г.М. О разностном аналоге неравенства $\|p\|_{L^{2}} \leqslant$ $C\|\operatorname{grad} p\|_{W_{2}^{-1}}$. Препринт № 67. М.: ОВМ АН СССР, 1983.

14. Рид М., Саймон Б. Методы современной математической физики. Т. 1. М.: Мир, 1977.

15. Боговский M.E. Решение первой краевой задачи для уравнения неразрывности несжимаемой среды // ДАН СССР. 1979. Т. 248. № 5. С. 1037-1040.

16. Аәранович М.С., Вишик М.И. Эллиптические задачи с параметром и параболические задачи общего вида // УМН. 1964. Т. 19. № 3. С. 53-161.

17. Березанский Ю.М. Разложение по собственным функциям самосопряженных операторов. Киев: Наукова думка, 1965.

18. MacCamy R.C., Wong J.S.W. Stability theorems for some functional equations // Trans. AMS. 1972. V. 164. P. 1-37.

19. Владимиров В.С. Уравнения математической физики. М.: Наука, 1988.

20. Эдвардс Р. Ряды Фурье в современном изложении. Т. 1. М.: Мир, 1985.

21. Люстерник Л.А., Соболев В.И. Краткий курс функционального анализа. М.: Высш. школа, 1982.

22. Мазья В.Г. Пространства Соболева. Л.: Изд-во ЛГУ, 1986.

Поступило в редакцию 27.IV.1995 